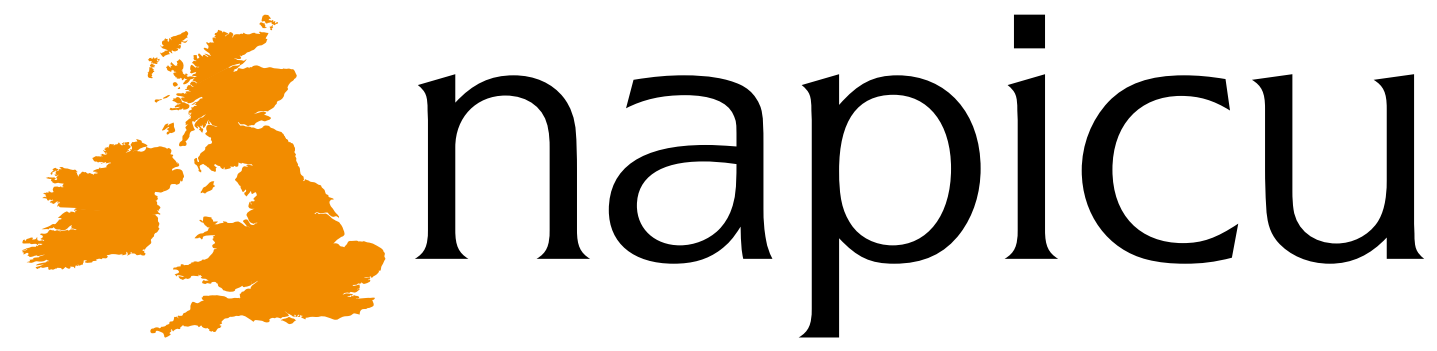

national association of psychiatric intensive care $\varepsilon$ low secure units

\title{
National Minimum Standards for Psychiatric Intensive Care in General Adult Services
} Updated 2014 


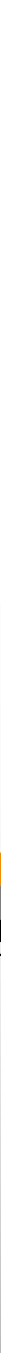




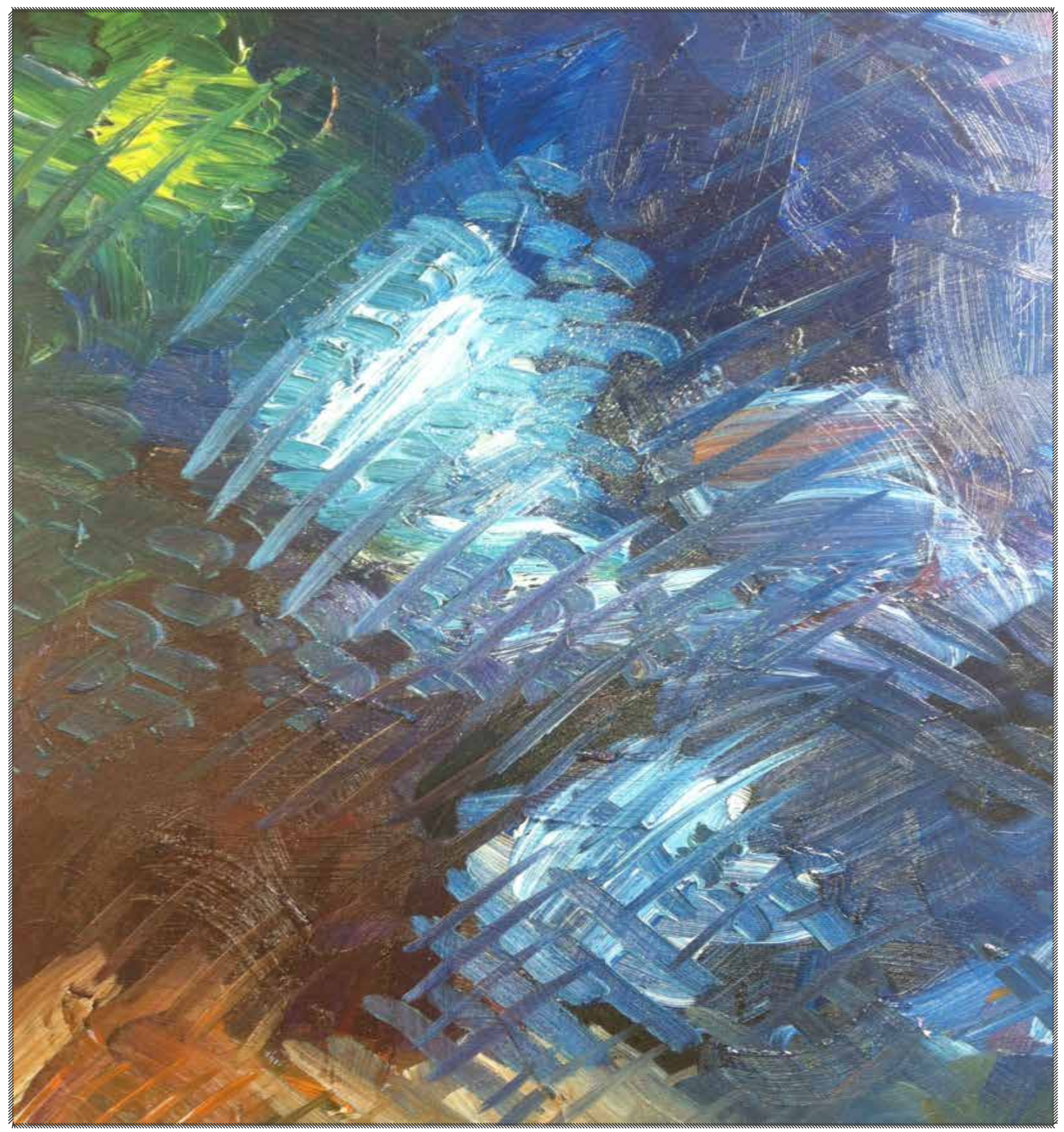

'New Dimensionalism' Anonymous Acute Care Patient (2012) 


\section{National Minimum Standards for Psychiatric Intensive Care in General Adult Services, Updated 2014}

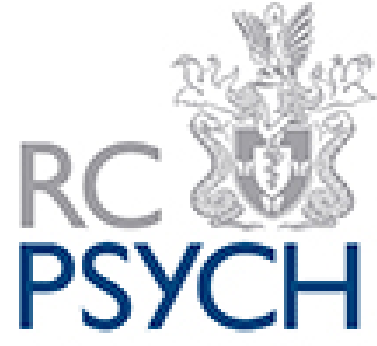

ROYAL COLLEGE OF PSYCHIATRISTS

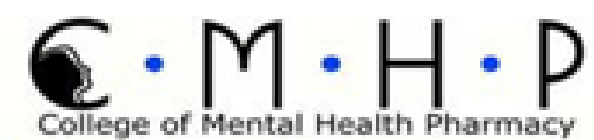

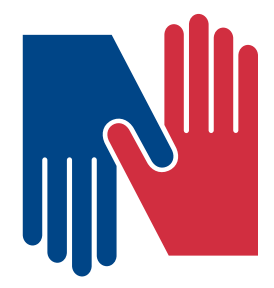

Royal College of Nursing

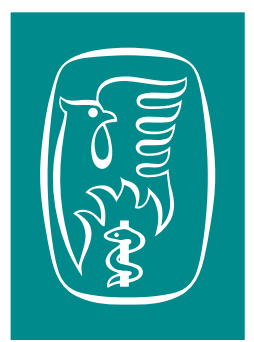

College of Occupational Therapists 


\section{Contents}

ABBREVIATIONS

FOREWORD 1

INTRODUCTION 4

1. DEFINITIONS 5

2. ADMISSION CRITERIA 6

3. CORE INTERVENTIONS 10

4. MULTIDISCIPLINARY TEAM SERVICE STRUCTURE AND PERSONNEL 16

5. OPERATIONS AND CLINICAL LEADERSHIP 19

6. PICU CARE PATHWAYS 22

7. PHYSICAL ENVIRONMENT 25

8. PATIENT INVOLVEMENT 32

9. CARER INVOLVEMENT 35

10. DOCUMENTATION 37

11. ETHNICITY, CULTURE AND GENDER 39

12. SUPERVISION 41

13. LIAISON WITH OTHER AGENCIES 43

14. POLICIES AND PROCEDURES 44

15. CLINICAL AUDIT AND MONITORING 47

16. STAFF TRAINING 50

17. CONTINUING PROFESSIONAL DEVELOPMENT 53

18. SECURITY AND RISK ASSESSMENT 55

19. PICU SUPPORT INFRASTRUCTURE 58

20. OTHER TYPES OF PSYCHIATRIC INTENSIVE CARE 59

21. STAFFING LEVELS 63 REFERENCES 66

ACKNOWLEDGEMENTS 70

APPENDIX 1: STAFF TRAINING 71

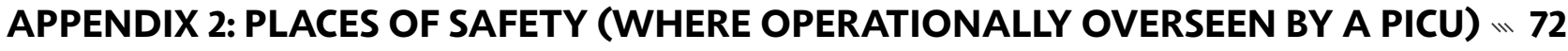





\section{Abbreviations}

BPRS Brief Psychiatric Rating Scale

CAMHS Community \& Child Adolescent Mental Health Services

CMHT Community Mental Health Team

CPA Care Programme Approach

CPD Continuing Professional Development

CPS Crown Prosecution Service

CQC Care Quality Commission

ECA Extra Care Area

ECT Electroconvulsive Therapy

G-CSF Granulocyte-Colony Stimulating Factor

HoNOS Health of the Nation Outcome Scales

LUNSERS Liverpool University Neuroleptic Side-Effect Rating Scale

MAPPA Multi-Agency Public Protection Arrangements

MDT Multidisciplinary Team

MHA 1983 Mental Health Act, 1983

NAPICU National Association of Psychiatric Intensive Care and Low Secure Units

PANSS Positive and Negative Syndrome Scale - Standard Version

PICU Psychiatric Intensive Care Unit

PoS Place of Safety

RC Responsible Clinician

RFID Radio Frequency Identification

RT Rapid Tranquillisation

SCIP Strategies for Crisis Intervention and Prevention

SOP Standard Operating Procedure 
I am delighted in my final days as President of the Royal College of Psychiatrists to have been asked to write the Foreword to this important update of the National Minimum Standards for Psychiatric Intensive Care in General Adult Services.

These clinical standards for psychiatric intensive care units (PICUs) are a revision of the 2002 National Minimum Standards, and reflect the fact that over the past 12 years, psychiatric intensive care units have further evolved to become a core component of the acute inpatient care pathway. Furthermore, the model of care in the general adult PICU has led to innovation in other PICU types, such as the medium secure PICU, the child and adolescent PICU and women's PICU.

PICUs manage some of the most clinically unwell and acutely risky patients; in this clinical area, it is easy to think that patient and carer perspectives have a secondary role. I therefore very much welcome the fact that this document, like the 2002 National Minimum Standards, emphasises the key role of patient and carer involvement and psychological interventions in achieving successful outcomes in the PICU. It goes without saying that we absolutely have to get care right when people are at their most unwell; documents like this, setting out clearly agreed standards for care, can help. I also welcome the emphasis on audit, supervision and a structured approach to giving care.

The strength of these standards is, ultimately, derived from their multidisciplinary focus, and by putting the clinical need of the patient at the heart of service delivery.

The standards contain a clear definition of psychiatric intensive care, as distinct from low secure care. This is not surprising given the move of low secure care to a more forensic focus, and the new landscape of commissioning. More importantly, it shows that PICU has developed as a speciality over the past 12 years, and there is much more clarity about the position of PICU in the inpatient care pathway, the nature of PICU patient types, and the core interventions provided by the PICU.

The PICU manages the most acutely unwell patients in the inpatient care pathway, and this can only be done well with an engaged multidisciplinary team, with clear clinical and operational leadership at multiple levels. This is required at multiple levels, from the management of challenging clinical scenarios, to the operation of the PICU, to the integration of that PICU within the wider system. It is refreshing to see that the document looks at leadership from the multidisciplinary angle, whilst still retaining an overall clarity of vision.

The National Association of Psychiatric Intensive Care Units (NAPICU) has for years been leading in this clinical area, and as a national association, its hallmark is that it is executive leadership and its membership are multidisciplinary. These standards reflect this fundamental attribute of high-quality clinical care, and also cite all clinical and non-clinical disciplines which have a role in the management of PICU patients.

The PICU movement over the past two decades has propelled the long-standing adage that better physical inpatient environments provide a vehicle for more therapeutic care. This set of revised standards takes this head-on and should allow PICUs to continue leading in this area. The science behind the design of inpatient environments needs a catalyst, and PICUs have been innovating in this area for the past decade, so are well placed to take this further in the next decade.

Importantly, this document recalibrates the balance between risk management (security) and therapeutic interventions in the PICU, by introducing a four-dimensional security matrix. It essentially states that the treatment model - one of intensive and dynamic clinical risk assessment and management - is integral to maintaining safety in the PICU. 
One of NAPICU's aims is to support and develop staff working in PICUs. I am delighted that this revised document raises the profile of this important issue. In an era where staff training and development can be somewhat forgotten, these standards remind us that inpatient psychiatric specialisms (of which PICU is but one) require specific skills, training and support. Better skilled, better trained and well supported clinicians will form better teams, and provide a higher quality of clinical care.

During my time as President of the RCPsych I have had a particular focus on working to achieve parity for mental health. This has included the need to ensure that mentally ill patients also receive high-quality physical healthcare, and this is an area in which I will continue to have an important role, as Chair of the Academy of Medical Royal College's Health Inequalities Forum. Many patients in PICUs are not just psychiatrically unwell, but have significant co-morbid physical health problems. These standards rightly give high priority to the management of physical health problems in the PICU, and its model of care places this on an equal footing with mental healthcare. This is a template to which we should all aspire.

The next decade promises to be even more exciting for this area of clinical care, with the PICU model being considered in other patient groups, and the beginnings of the desire to underpin PICU practice with a valid and reliable evidence base.

These standards provide a framework to consider the challenges which lie ahead, and will provide an invaluable steer for patient groups, clinicians, managers, researchers and commissioners as they navigate their way through these challenges.

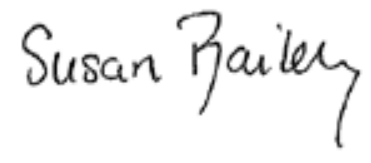

Professor Dame Sue Bailey, OBE, FRCPsych President, Royal College of Psychiatrists

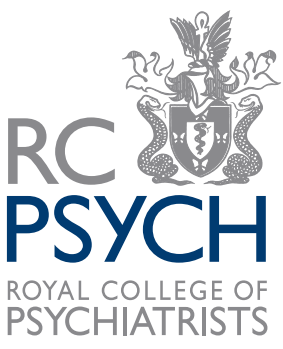

June 2014 
I am delighted as Chief Executive and General Secretary of the Royal College of Nursing to offer a foreword to this update of the National Minimum Standards for Psychiatric Intensive Care in General Adult Services.

This document, like the service it describes, was developed in the context of a multidisciplinary team of professionals and the individuals (both patient and carers) that it will serve. It is striking in that the time that has lapsed since the original standards were published in 2002 that such an approach is no longer novel but to be expected, if not demanded, in today's environment of co-production.

I know from my own visits to clinical areas that it is even more important than ever that teams of health professionals faced with the demands upon the service they deliver work effectively as a team. This requires not only strong and purposeful leadership but a team that is committed to providing the highest standards of care. We know that in environments such as those described in these standards that the needs of patients are not only more acute as presentations change but that the demands upon the service are similarly acute. It is all the more important to have a clear vision and understanding of the standards that such a service must aspire to achieve.

In an environment where care and security must by necessity co-exist, the specialised skills of professionals working in such an environment are recognised in these standards. I am particularly pleased to see the recognition given to the need for ongoing support and development of all staff working in the service. We have a long held conviction that investment in the people who provide a service is a wise investment to assure the outcomes that all wish to achieve.

NAPICU is to be congratulated for its vision of how a service to the most unwell, and at times risky, clients is to be articulated. This includes not only the physical environment, but also the central role of the evidence based interventions that are utilised by professionals to achieve the best outcomes for those in receipt of the service.

There is no doubt that services such as those provided in PICU's face a difficult and challenging future. Central to the success of these services will be the ability to articulate their worth in contributing to the welfare of those they serve. This document clearly realises that ambition.

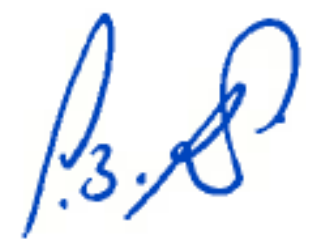

\author{
Dr Peter Carter, OBE \\ Chief Executive and General Secretary of the Royal College of Nursing
}


The National Association of Psychiatric Intensive Care Units (NAPICU) was established in 1996 to provide guidance on psychiatric intensive care issues in the UK, to overcome variability of practice and terminology where definitions of units are concerned and also to ensure high quality multidisciplinary care.

The aims of NAPICU are as follows:

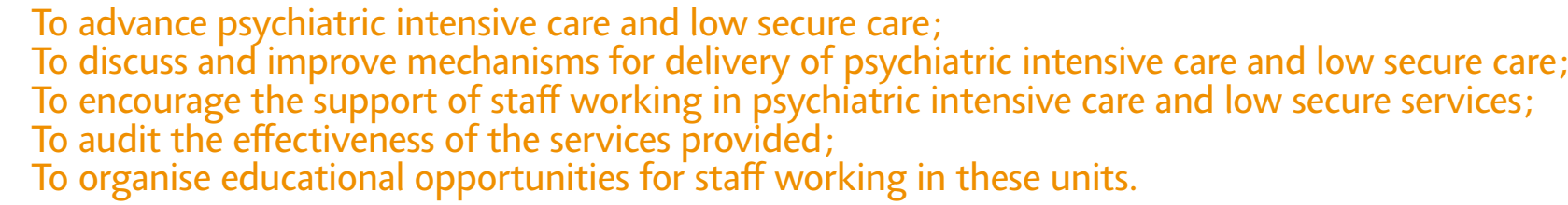

A PICU policy, research and development group was established at the North East London Foundation Trust by $\mathrm{Dr}$ Stephen Pereira in 1999. This group conceptualised and initiated a project to develop the National Minimum Standards in psychiatric intensive care and low secure environments given the absence of such national standards in the UK. A multidisciplinary group of clinicians in the field of psychiatric intensive care and low secure care was established and produced clinical standards, which were subsequently adopted by the Department of Health in 2001. The National Minimum Standards in psychiatric intensive care units and low secure environments was launched as a policy implementation guide in 2002 (Pereira \& Clinton, 2002). This group also initiated the PICU and low secure practice development network to help units to meet with the national standards. The main aim of the 2002 National Minimum Standards was to develop standards of psychiatric intensive care and low secure environments with a multidisciplinary and patient focus. The National Minimum Standards have influenced the successful development of psychiatric intensive care units and low secure environments in a standardised manner across the UK, whilst allowing for local creativity and innovation ultimately leading to high quality care for patients who find themselves in these environments.

Over the last ten years, the 2002 National Minimum Standards have been the framework for developing high quality PICU and low secure services. PICU and low secure services across the UK have been on a developmental journey with the sole aim of providing more efficacious, safer and holistic care for our patients, by a well-trained and motivated clinical team in a clinical environment that is more than just fit for purpose. Psychiatric intensive care has developed as a speciality in its own right, and PICUs are found across the mental health service structure, in clinical services traversing the life-span, across mental health security levels, and in a range of clinical specialities. Low secure services have also undergone a transformation with other variants emerging over the past ten years, incorporating elements of security, rehabilitation, challenging behaviour and treatment resistance models.

In 2012, NAPICU worked with the Department of Health to produce two draft good practice commissioning guides (Department of Health, 2012a, b) in the clinical areas of psychiatric intensive care and low secure care. This process highlighted a need to revise the clinical standards set in 2002 to reflect the current clinical processes in PICU and low secure care, and to set the clinical framework for the coming decades in this challenging area.

This document looks at the clinical standards in PICU services. The project group was set up through the NAPICU executive, a group which comprises multiple professional disciplines, patient and carer representatives, and expertise in all types of PICU modality.

The remit of the group was to revise the agreed standards for psychiatric intensive care services and the general good practice guidance for each of these standards. The overall objective is to provide patients, carers, clinicians, managers and commissioners with a dynamic framework for delivering high quality psychiatric intensive care services. These standards are derived from the clinical perspective, which in turn is driven by the achievement of a positive and empowering experience for patients in psychiatric intensive care units.

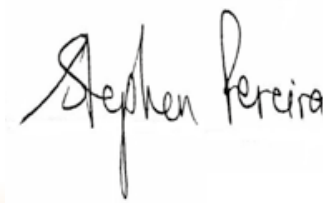

Dr Stephen Pereira Chairman NAPICU

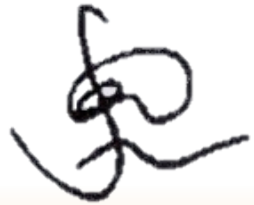

Dr Faisil Sethi

Vice-Chairman NAPICU 


\section{Definitions}

\subsection{Introduction}

It is important that PICUs are clearly defined in order to ensure clarity of purpose and quality of care. Since the publication of the first edition of these standards (Pereira \& Clinton, 2002), PICU and low secure provision have been established as specific nationally recognised clinical facilitates that should not be confused with other locally derived approaches sometimes deployed in acute mental healthcare. Some examples of terms assigned to these local facilities include 'high dependency unit', 'high care area', 'intensive care area of acute ward', 'locked acute ward' and 'acute admissions unit'. These facilities are often poorly evaluated and are inconsistent in terms of their purpose, infrastructure and efficacy. Only units meeting these standards should be considered as PICUs. Access to a recognised PICU is required for all acute mental health services.

\subsection{Psychiatric intensive care}

Psychiatric intensive care is for patients who are in an acutely disturbed phase of a serious mental disorder. There is an associated loss of capacity for self-control, with a corresponding increase in risk, which does not allow their safe, therapeutic management and treatment in a less acute or a less secure mental health ward. Care and treatment must be patient centred, multidisciplinary, intensive and have an immediacy of response to critical clinical and risk situations. Patients should be detained compulsorily under the appropriate mental health legislative framework, and the clinical and risk profile of the patient usually requires an associated level of security.

Psychiatric intensive care is delivered by qualified and suitably trained multidisciplinary clinicians according to an agreed philosophy of unit operation underpinned by the principles of therapeutic interventions and dynamic clinically focused risk engagement. Length of stay must be appropriate to clinical need and assessment of risk, but would aim not exceed eight weeks in duration.

\subsection{Low secure care}

Low secure care is for patients who present with more prolonged disturbance or complex needs in the context of a serious mental disorder, requiring a 'low secure' level of physical, relational and procedural security. This may be due to an objective risk to others or because of associated criminal justice requirements. Low secure care is positioned between mainstream general adult psychiatric settings and forensic medium secure settings.

In general, there are three distinct patient types who may require low secure care, these are:

- Those who require a step-down in security from high or medium secure;

- Those subject to requirements emanating from the criminal justice system;

- Those who require rehabilitation due to prolonged challenging behaviour, treatment-resistance and associated risk consistent with the need for low security.

Care and treatment must be patient centred, multidisciplinary, primarily rehabilitation and recovery-focused, and able to respond to critical clinical and risk situations. Patients should be detained compulsorily under the appropriate mental health legislative framework including parts II and III of the Mental Health Act (MHA 1983).

Low secure care is delivered by qualified and suitably trained multidisciplinary clinicians according to an agreed philosophy of unit operation underpinned by the principles of clinically focused risk management. Length of stay must be appropriate to clinical need and assessment of risk, and can generally range between six months and two years. 


\section{Admission criteria}

\subsection{Rationale}

Admission and detention in a locked environment constitutes a fundamental loss of freedom for an individual. PICU staff need to work collaboratively with referring services to ensure that admission is appropriate to the individual's needs. There should be no more restrictions on a person's freedom than is warranted by his or her clinical condition.

The following describes the general criteria for patients who will be admitted to the PICU. The admission criteria will form the basis of the unit's admission policy. Furthermore, they can be distributed to the PICU referral agencies to act as a reference source, informing those who may be considering making a referral.

\subsection{Standard}

\section{Behaviour requiring PICU}

2.2.1. The PICU has a primary role in helping patients (and mental health services) successfully overcome the challenges and risks presented by disturbed behaviour. Whilst much of the rationale for admission to the PICU can be measured in behavioural terms, it is also accepted that assessed increased need, or risk, as a result of acute symptoms may also form a reasonable basis for PICU admission.

2.2.2. Patients admitted to the PICU should exhibit mental state or clinical behaviour which seriously compromises their physical or psychological well-being, or that of others, and which cannot be safely assessed or treated in a general adult ward facility.

2.2.3. The behaviour that results in the need for admission to the PICU will be as a direct result of an acute mental health condition, or an acute exacerbation of and existing mental disorder, which is expected to respond to the treatment approaches available to the PICU in a relatively short period of time.

2.2.4. Mental and behavioural characteristic requiring PICU admission will generally fall under one, or more, of the following headings (Dix, 1995):

- Externally directed aggression. A patient is assessed as posing a significant risk of harm to others, or extreme aggression towards property;

- Internally directed aggression. A patient is assessed as posing a significant risk of suicide and the patient is unresponsive to preventative measures available;

- Absconding. Patients who are detained under the MHA 1983, for whom the consequences of persistent absconding are serious enough to warrant treatment in the PICU. The PICU should not provide security for its own sake, there should always be a primary clinical reason for admission to prevent absconding;

- Unpredictably. Unpredictable patients, potentially posing a significant risk to self or others and requiring further assessment.

\section{Non-emergency planned admissions for intensive therapeutic interventions}

2.2.5. While all PICU admissions should involve the setting of treatment goals, PICUs should also potentially be able to offer admission outside the context of an acute emergency situation. 
2.2.6. A planned admission to the PICU will be negotiated with the multidisciplinary team (MDT) in advance of admission according to specifically identified treatment needs and goals. The increased potential for engagement, the improved patient to staff ratio and a more controlled environment provides increased opportunity for the PICU to meet specific treatment goals.

2.2.7. The basis for referral to the PICU for a planned admission should include the following:

- The patient has experienced significant difficulty in regaining their potential for functioning and also presents significant difficulties or concerns within their current placement;

- The referral for planned admission is supported by the patient's current MDT and where possible the patient themselves;

- The referral includes predefined and agreed goals for the admission between the referring MDT, the PICU and where possible the patient themselves;

- The treatment goals, if achieved, are expected to have sufficient positive impact to justify transfer to the PICU;

- The period of time expected to achieve the treatment goals is agreed in advance between the referring MDT the PICU and where possible the patient.

\section{Admission process}

2.2.8. The specific reasons for admission should be agreed between referrer and assessing team. This should be documented clearly in the referral to PICU form, outlining the purpose of admission and high level treatment objectives. These objectives should form part of the care plan in PICU admission and the demonstrable clinical rationale for admission.

2.2.9. Patients should only be admitted if they display a significant risk of aggression, absconding with associated serious risk, suicide or vulnerability (e.g. due to sexual disinhibition or over activity) in the context of a serious mental disorder.

2.2.10. Admission to a PICU should be due to a new episode or to an acute exacerbation of the patient's condition which cannot be safely managed in a general adult ward. In contrast, patients in low secure units may be experiencing chronic behavioural disturbance not necessarily due to an acute clinical presentation.

2.2.11. It should have been demonstrated that multidisciplinary management strategies in the referring acute admission unit have not succeeded in containing the presenting problems. Initial management strategies attempted should be outlined clearly in the referral to PICU if referred from another treatment setting within mental health (e.g. acute wards).

2.2.12. There should be mutual agreement between referrer and admitting unit on the positive therapeutic benefits expected to be gained from the time-limited admission, including a clear rationale for assessment and treatment.

2.2.13. Where possible, all patients referred to the unit should have an assessment of their needs and risks undertaken by members of the PICU team who make a decision to admit, prior to the patient arriving in the unit (Cresswell et al. 2010).

2.2.14. While historical factors will play an important part in assessment, current symptomatology should be the prime consideration in determining whether admission is appropriate. 
2.2.15. If admission is not thought to be appropriate, PICU staff should be able to offer advice and guidance on the management of the patient. In terms of support for patient management, PICU staff should be available for follow-up advice and management as needed and any subsequent re-assessment if escalation of clinical need occurs.

2.2.16. Where a patient is being admitted directly from the community, the admitting staff should check that the referring agency has given clear information as to the security of the patient's home, whereabouts of children or animals etc.

2.2.17. All PICUs should develop standardised, robust risk assessment procedures; initiated as part of preadmission. These should include objective validated measures as well as comprehensive, multidisciplinary, clinical risk assessment systems (see Chapter 18).

2.2.18. There should be an information-sharing protocol regarding clinical environments (specifically PICU in this instance) whose purpose is confirmed at organisation board level and of which all staff are aware; this should be publicised to visitors and patients in a variety of media and languages (see 14.2.1.9).

2.2.19. The PICU should have an operational policy document that outlines the type of patient who should be referred to receive treatment within such an environment (this should be based upon these standards). The policy document should be agreed within the organisation and publicised to referring wards and agencies (see Chapter 6 \& Chapter 14).

2.2.20. Managers and practitioners should have written standards for the admission process.

\section{Adult PICU inclusion criteria}

2.2.21. Patients admitted should be aged 16 or over ( 18 or over if in full-time education). Patients should not normally be over the age of 65 ; exceptions to this are specifically related to physical state and primary condition which does not conflict with exclusion criteria (see 2.2.28).

2.2.22. Patients should be detained under the appropriate completed assessment/treatment section (not admitted under Section 136) of the MHA 1983.

2.2.23. Assessment by members of the PICU MDT should occur prior to admission. The assessment should include objective measures and be clinically robust.

2.2.24. Restricted patients (those subject to restrictions under the MHA 1983, via the courts, prisons or Ministry of Justice) should not be accepted unless there is clear pathway or provision to transfer them to a more appropriate clinical environment as warranted by the clinical or risk profile (e.g. a medium secure unit, a low secure unit, a general adult psychiatric inpatient ward or a criminal justice setting) if warranted by their clinical condition.

2.2.25. Patients with specialist conditions which may not be primarily psychiatric in nature but do have associative symptomatology, clinical or risk profile, should not be accepted unless there is clear pathway or provision to quickly transfer them to a more appropriate clinical environment (e.g. Huntington's Disease.)

2.2.26. The admission of young people to an adult ward must meet the age appropriate guidance published by the Department of Health. 
2.2.27. Children under the age of 16 should not be admitted to an adult PICU. Patients between the ages of 16 and 18 who are in full time education should not be admitted to an adult PICU.

\subsubsection{Admission should not occur in the following circumstances:}

- The patient is assessed as presenting too high an internal or perimeter security risk for the PICU type, requiring medium or high secure PICU;

- The patient has a primary diagnosis of substance misuse and the primary purpose of admission is solely to prevent access to substances;

- The patient's behaviour is as a direct result of substance misuse and they are not suffering from an exacerbation of their mental disorder at the time of referral;

- The patient has a primary diagnosis of dementia;

- The patient has a primary diagnosis of Learning Disability (LD) and requires a specialist LD facility;

- The patient's physical condition is too frail to allow their safe management in a PICU;

- The patient has a chronic condition which would not benefit from admission to PICU;

- The patient is restricted (subject to restrictions under the MHA 1983, via the courts, prisons or Ministry of Justice) and has no clear pathway or provision for transfer from the PICU once clinically warranted;

- Admission would mix the gender of the patient population unless separate sleeping areas and day areas (Department of Health, 2010a) are available.

\subsection{Good practice guidance}

2.3.1. A decision to treat a patient within a PICU should only be made when clinically warranted by the degree of presentation and associated clinical risks, and when there are identified limitations in the treatment capability of the referring clinical setting (Department of Health, 1999).

2.3.2. A clear purpose of admission to PICU should be understood universally within the acute care pathway (see Chapter 6 ) and be supported by clear referral mechanisms and clinical support structures. An estimated date for transfer back to less intensive provision should be considered at the earliest opportunity, which may be during the admission process.

2.3.3. Clear inclusion and exclusion criteria for admission to the specialist environment of PICU should be adhered to, and a procedure for considering differences of opinion established.

2.3.4. Psychiatric disorders are more prevalent in adults with LD (Deb et al. 2001); between 20.1 per cent to 22.41 per cent compared to 16 percent in the general population (Cooper et al. 2007). The Equality Act places a statutory responsibility on all organisations to make 'reasonable adjustments' to avoid discrimination (Equality Act 2010). However there remains debate as to whether the psychiatric care for people with learning disabilities, and particularly those who present with co-morbid mental illness should, as championed within Valuing People (Department of Health, 2001) be undertaken by mainstream services as per the normalisation agenda, or whether the specific needs of such a complex group preclude their effective care and treatment outwith specialist services.

2.3.5. When people with LD are admitted to acute adult inpatient services there are clear risks that they may be vulnerable, marginalised, and their admission may be prolonged (Bouras et al. 2004). There are a group of LD patients for whom their involvement with secondary and tertiary inpatient services, and whilst complicated by their LD, is triggered by acute mental illness. Where their level of need meets the necessary criteria, admission to a PICU service will be wholly appropriate. Such admissions should be as brief as possible, this can be helped by appropriate staff awareness training, good liaison between inpatient and community services, up to date health action plans, access to advocacy services and support from community LD assessment and/or treatment services. Support will be particularly relevant for individuals with adaptive or language deficits or an underlying pervasive developmental disorder (NDTi 2012). 


\section{Core interventions}

\subsection{Rationale}

The PICU should ensure that patients are provided with an intensive, multidisciplinary, therapeutic program and evidence-based interventions whilst admitted to the unit. These interventions should be directed towards rapidly reducing acute symptoms whilst promoting self-management and recovery. Interventions must be provided by appropriately qualified and trained individuals (see Chapter 4).

\subsection{Standard}

\section{Physical health}

3.2.1. Specific expertise will be available to lead on physical healthcare assessment and management.

3.2.2. Provision should be made for physical health examinations, relevant investigations (including imaging), and the management of any adverse medication effects. This should be overseen by senior medically qualified staff.

3.2.3. Staff carrying out physical examinations should either be of the same gender, a same-sex chaperone should be present, or the patient should be able to choose the gender of the staff member.

3.2.4. This involves liaison with medical colleagues in other specialities to ensure parity between physical and mental healthcare outcomes.

\section{Risk assessment and management}

3.2.5. Assessment and management of risk is central to effective and safe care, as is formulating management plans to minimise the risk.

3.2.6. The PICU should keep, maintain and update the skills required to implement a number of evidence based risk assessment tools relevant to a number of risk domains including:

- Violence and aggression

- Suicide and self-harm

- Vulnerability

- Potential for offending in the context of mental disorder e.g. sexual risk, fire setting, etc.

3.2.7. Risk assessments should include consideration of adult safeguarding, child protection, victim considerations, and other vulnerabilities (for example alcohol and drug use, financial exploitation).

\section{Assessment and diagnosis}

3.2.8. Provision should be made for the regular assessment and recording of mental state examinations and behaviour by appropriately qualified professionals.

3.2.9. Consultant psychiatrists and other medical staff should ensure the application of medical knowledge and clinical skills against a backdrop of evidence-based practice (Royal College of Psychiatrists, 2010). 
3.2.10. In collaboration with the MDT, the medical team should lead on formulating, devising and reviewing diagnoses. This includes combining biological, occupational, psychological, social and cultural factors, whilst keeping in mind any associated physical health disorders.

\section{Communication}

3.2.11. There should be activity for the development of therapeutic relationships between unit staff including appropriately qualified professionals able to engage patients in psycho-education.

3.2.12. Responsible individuals and a clear process for family and carer liaison should be established (see Chapter 9).

3.2.13. Effective communication should take place with other agencies and stakeholders in the PICU care pathway to facilitate the patient journey through the system (see also 5.2 \& Chapter 11).

\section{Treatment - pharmacological}

3.2.14. The medical team are primarily responsible for the prescribing and monitoring of medications. This also includes reviewing adverse effects and dosages to ensure optimal outcome for the patient.

3.2.15. Polypharmacy and the use of above BNF (British National Formulary) dosages of medications should be avoided as far as possible. However, when they are used, there must be a clear documented rationale by a specialist psychiatrist or pharmacist; there should be regular review of such practice.

3.2.16. PICU professional staff should ensure that there is a defined and well understood procedure for recognising and closely monitoring any side effects from prescribed medication. The PICU should keep, maintain and update skills required to implement a number of evidence-based, validated side effect assessment scales (either clinically assessed or self-reported).

3.2.17. A specialist mental health pharmacist working in the PICU should have a good understanding of the general pharmacological management of the acute presentations of mental disorder and acute disturbance.

3.2.18. A specialist mental health pharmacist should work with other professionals in the PICU to ensure a high standard of medicines management.

3.2.19. Appropriately qualified and senior medical staff should lead ongoing formulation of a pharmacological treatment plan. This should occur with input from patients and the MDT.

3.2.20. There should be an active review of all prescribed medicines at least once a week in the MDT review meeting with the consultant psychiatrist and other members of the MDT, ensuring that those who need medicines get optimal regimes.

3.2.21. There should be provision for a specialist mental health pharmacist to visit the unit several times a week and take part in the weekly MDT reviews.

3.2.22. Many medicines will need to be reviewed on a daily basis, and the pharmacist needs to visit the PICU frequently enough to do this with prescribing colleagues, focusing on their therapeutic as well as adverse effects. 
3.2.23. All prescriptions for medicines should be 'clinically screened' by a pharmacist to ensure suitability, safety, and that the relevant legal requirements are followed.

3.2.24. Pharmacists should assist prescribers with the medicines reconciliation process on admission of the patient to the PICU.

3.2.25. Where appropriate, pharmacists and prescribers should use therapeutic drug monitoring to safely optimise doses of specific medicines.

3.2.26. All patients should have access to independent education and advice about medicines, preferably from a specialist mental health pharmacist in the PICU.

3.2.27. The advice of a specialist pharmacist should be available to other staff regarding the administration and storage of medicines on the unit.

3.2.28. The input of the specialist mental health pharmacist in the PICU will be required in treatmentresistant and challenging cases, to work alongside the MDT and review the prescribing history. Such reviews should lead to individualised pharmacological treatment plans which balance clinical need and risks, whilst enhancing patient choice.

3.2.29. Mental health nurses in particular should be able to offer patients and carers written and verbal information about their medications (for both their physical health and mental health disorders). This should be delivered in conjunction with medical and pharmacy colleagues in the PICU.

3.2.30. The PICU medical team should have access to a wide range of treatment interventions for the management of acute mental disorder presentation. This should include the access to a general medical hospital setting and electroconvulsive therapy (ECT). ECT must be given in line with current best practice guidance (e.g. Waite \& Easton, 2013; NICE, 2003).

\section{Rapid tranquillisation (RT)}

3.2.31. RT can be safely used to control acutely disturbed and/or violent behaviour when other nonpharmacological methods of de-escalation have failed or are inappropriate (NICE, 2005; Department of Health, 2014). All relevant monitoring equipment and medicines to manage any adverse effects should be readily available in the unit (NICE, 2005).

3.2.32. The medical team should be responsive to the acute and changing needs of the patient receiving treatment in the PICU. This includes the management of acute disturbance with interventions such as RT.

3.2.33. There should be a robust protocol regarding RT usage which is consistent with national guidance (e.g. NICE, 2005) including relevant physical monitoring of patients.

3.2.34. The use of RT should be monitored regularly and audited at least annually.

\section{Overall therapeutic engagement er activity}

3.2.35. Each patient should be invited to meet with a member of PICU staff for one-to-one contact each waking shift and this should be documented.

3.2.36. All patients should have a daily multidisciplinary review directly involving at least the medical and nursing clinicians in the PICU. 
3.2.37. All patients should receive an in-depth multidisciplinary review at least once a week, involving the whole MDT and external professionals, which incorporates the views of carers. All patients must have a faceto-face review with a consultant once a week (NICE, 2012).

3.2.38. The MDT should obtain details of the family and social circumstances prior to admission.

3.2.39. Liaison with the patient's care co-ordinator or community mental health team (CMHT) should occur in the first week of admission to a PICU. This should be repeated at regular intervals during the PICU admission.

3.2.40. Boundary setting interventions should be utilised for the shortest duration possible in a therapeutic manner. This may include contracting, de-escalation, restraint, time-out and seclusion. Developing strategies for understanding the reasons and context for disturbed behaviour should be a core function of the PICU. Any interventions engaging disturbed behaviour should pay careful attention to the evidence and national best practice guidance such as "Positive and Proactive care: reducing the need for restrictive interventions" (Department of Health 2014).

3.2.41. Overall engagement should be underpinned by an emphasis on coping strategies to self-manage acute symptoms where possible.

3.2.42. Health promotion activities should be available and encouraged, including: diet; exercise; substance misuse and smoking cessation.

3.2.43. Social-skills or life-skills training should be available, incorporating psycho-education, on topics relating to activities of daily living such as interpersonal communication, relationships, coping with stigma and stress management.

3.2.44. The unit should have access to interpreters, sign language and other communication media as appropriate (see Chapter 11).

3.2.45. Following risk assessment, patients should be able to leave the unit to attend activities elsewhere in the building and, with appropriate supports and escorts, to access usable outdoor space every day, where detention status permits.

3.2.46. Staff with appropriate expertise in social service processes should be actively involved in the provision of social work assessments, Care Programme Approach (CPA) meetings and community care planning.

3.2.47. The PICU team should ensure that a defined activity programme is available to all patients, with individual and tailored activities where required. This should facilitate meaningful occupational choice and be available at weekends and evenings (College of Occupational Therapists, 2012).

3.2.48. PICU professional staff should ensure the assessment of mental health symptomatology including impairment of concentration, perception and orientation.

3.2.49. There should be provision for specialist functional assessments by a specialist occupational therapist using standardised tools; for example: Assessment of Motor and Process skills (AMPS); Assessment of Communication and Interaction skills (ACIS) Canadian Occupational Performance Measure (COPM); Model of Human Occupation Screening tool (MOHOST) (College of Occupational Therapists, 2012); and assessments to aid diagnosis (for example behavioural memory testing batteries, such as the Rivermead (Wilson et al. 2008).

3.2.50. Models of occupation should be used to ensure consideration of the occupational life history of patients and their past, present and future roles; for example, the Model of Human Occupation (MOHO) 
or the Canadian Model of Occupational Performance (CMOP)(College of Occupational Therapists, 2012). 3.2.51. PICU professional staff should ensure the delivery of psycho-education to deconstruct complex tasks, with the objective of promoting recovery and improved functioning.

3.2.52. Arrangements should be in place for appropriately qualified and trained staff to deliver group-work, individual skills sessions and activities of daily living (ADL).

3.2.53. The PICU MDT should ensure provision of a tailored and bespoke treatment, engagement and activity programme utilising the skills of all members of the MDT. This should have PICU specific consideration including functional, diversional, psycho-educational therapy focusing on the holistic care of acutely disturbed patients.

3.2.54. Patients should be assisted in the development of coping strategies and trigger-recognition, leading to an overall Wellness Recovery Action Plan (WRAP).

3.2.55. A range of art therapy and creative media should be available.

\section{Psychological interventions}

3.2.56. Patients should have access to specialist practitioners of psychological interventions for a minimum of one day (eight hours) per week per unit.

3.2.57. Each PICU should have an attached clinical psychologist with expertise in the clinical assessment and treatment of patients presenting with acute mental disorder and associated behavioural disturbance.

3.2.58. There should be specific consideration of psychological factors which may have triggered the acute presentation.

3.2.59. Psychological therapies should be available (both individual and group) to address immediate recovery needs.

3.2.60. Psychosocial interventions should be available for working with families aimed at education and support.

3.2.61. The psychologist should engage and empower patients to think psychologically in spite of their acute presentation, and motivate them to engage in medium to longer term psychological therapy where appropriate.

3.2.62. Psychological techniques (e.g. use of behavioural, psycho-therapeutic techniques) in the care plans for patients in the PICU should be supported by specialist advice.

3.2.63. Provision should be made for specialised psychological assessment of needs, abilities or behaviour (for example psychometric testing).

3.2.64. Psychological support should be provided for debrief following serious incidents and in the development of particular interventions involving physical restraint or seclusion.

3.2.65. Psychological thinking or mindfulness is a core characteristic of the whole PICU team, from interactions around medicines adherence to those around emotional support. Team-based interventions delivered by other professionals, such as nurses, will also focus on reflection and a psychological understanding of the challenges associated with managing acute disturbance (for example, reflective practice groups, case-based discussion). 


\section{Medico-legal}

3.2.66. All staff are accountable for relevant aspects of MHA 1983 paperwork including detention and capacity to consent to treatment. Robust and clear procedures are established to maintain and monitor compliance with the MHA 1983.

3.2.67. The PICU should have clear procedures for the provision of timely, accurate and detailed reports as required by mental health review tribunals, courts or other bodies.

3.2.68. The PICU should have reliable methods of ensuring the attendance of required staff at tribunals, hearing or court, to provide oral evidence in relation to the clinical condition, MHA 1983 and other issues.

\subsection{Good practice guidance}

3.3.1. The PICU professional team should ensure that their own knowledge and skills are up-to-date as required by the relevant professional bodies (e.g. Royal College of Psychiatrists, General Medical Council, Nursing Midwifery Council). Specialist PICU professionals have a core role in the teaching and training of all other members of staff, and PICU stakeholders.

3.3.2. Effective therapeutic communication between PICU staff and a patient should be a conscious, goaldirected process.

3.3.3. Clear and accessible records associated with the care planning process must be maintained to a high standard. Where at all possible, this should be completed in collaboration with the patient, carers and other members of the MDT.

3.3.4. PICU professional staff and their assistants should provide care and treatment to meet patients' physical, psychological, social, mental and spiritual care needs. This includes providing support and interventions to families, carers and friends who play a significant role in patients' lives in a way that supports their recovery.

3.3.5. PICU professional staff should be in the position to provide credible expert advice, underpinned by research evidence, to other clinical teams regarding the management of patients with acutely disturbed, challenging and aggressive behaviour.

3.3.6. PICU professional staff should ensure that accurate information is relayed to patients in a format that they are able to understand, thus empowering them to make informed choices as far as is practicable. Particular approaches to communicating information, known to be helpful to those with acute symptoms, should be available. These may include:

- Repeat discussions required where patients have poor concentration or diminished memory;

- Specifically created and clear documentation focused on priority areas;

- Specific assistance in clarifying and understanding existing information sources (e.g. leaflets, websites). 


\section{Multidisciplinary team service structure and personnel}

\subsection{Rationale}

The MDT, its make-up and functioning, are crucial factors in the provision of a safe, effective and quality service.

\subsection{Standard}

4.2.1. All PICUs should have input from professionals in the following disciplines within the context of core team skills:

- Medical (a consultant psychiatrist and a trainee psychiatric doctor)

- Nursing (a team of specialist inpatient nurses, both qualified nurses, healthcare support workers and assistant practitioners)

- Management (a manager and deputy with relevant clinical expertise)

- Occupational therapy (a specialist in psychiatric inpatient care)

- Psychology (a specialist clinical psychologist)

- Pharmacy (a specialist pharmacist with experience in acute psychiatric inpatient settings)

- Social work (access to a social worker to address holistic care needs)

- Activity, physical health and engagement (e.g. physiotherapist, sports therapist, dietician).

See Chapter 21 for further details.

\section{Shift staffing levels}

4.2.2. The unit should have an agreed core establishment, providing a minimum staffing level across all shifts. There should be systems in place to ensure that all factors affecting staffing numbers and skill mix are taken into consideration and staff levels are reviewed on a daily basis. The factors that affect staffing levels are:

- Therapeutic engagement

- Observation

- Staff capabilities (i.e. skill mix)

- Training

- Sickness and absence (including any loss of capacity due to responsibilities outside the PICU)

- Supervision

- Escorts

- Acuity levels

- Number of active (usable) beds within the PICU

- Bed occupancy

- Local human resource guidance.

4.2.3. A number of formulae have been developed to calculate minimum staffing levels within mental health inpatient environments.

4.2.4. It is important that there is agreement between front line clinical leadership and senior operational managers regarding safe minimum staffing levels to ensure quality of patient experience.

4.2.5. PICUs require proportionally higher staffing levels than would ordinarily be available in the general adult acute psychiatric ward. 
4.2.6. Nursing staffing levels in the PICU should not depend solely on the core establishment; clinical staffing numbers should take the following factors into consideration:

- Acuity levels;

- Bed occupancy;

- Clinical interventions such as need for higher observation levels;

- Tertiary factors such as sickness, training, leave and capacity which may be lost due to organisational responsibilities (e.g. staff involved in Root Cause Analysis Investigation).

For example, minimum shift nursing staffing numbers in a 10-bed PICU should not fall below six, six and four for early, late and night shifts respectively, but these numbers may vary based on the above factors. This number should not include management and other specific therapy staff.

4.2.7. Medical staffing levels in PICUs depend on a variety of factors, including the clinical characteristics of the patients, the size of the PICU, the acuity levels, the strength of PICU interfaces with acute wards and other services, and the associated responsibilities of the PICU consultant psychiatrist (in terms of other clinical roles, training, management and leadership).

4.2.8. The PICU should have dedicated consultant psychiatrist input, and at least one single dedicated subconsultant grade doctor for the unit.

4.2.9. Out-of-hours, the PICU should have access to medical input which can respond appropriately to urgent and critical clinical scenarios.

4.2.10. The PICU should be working towards a philosophy of providing responsive critical care 24 hours a day, 7 days a week.

\section{Leadership (see also Chapter 5)}

4.2.11. The PICU ward manager has a key role in clinical leadership, and this should also be a senior registered professional with significant inpatient experience and expertise. The PICU ward manager should take an equal share in the clinical leadership of the service in partnership with the lead consultant.

4.2.12. The PICU ward manager should have a particular focus on the overall team performance, ensuring the safe care of the patients, the quality of clinical approaches, and safety of the staff.

4.2.13. Each PICU should have its own dedicated lead consultant (medical or nursing) who should provide expert input into the clinical, managerial and operational matters relating to the PICU service.

4.2.14. The dedicated lead consultant for the PICU should be of a status and expertise equivalent to that of a clinician able to function as a responsible clinician (RC; within the meaning of the MHA 1983).

4.2.15. The dedicated lead consultant and ward manager should have the appropriate authority to lead the PICU and make decisions regarding all aspects of PICU unit operation. For many PICU operational issues, this requires the support of senior management within the mental health organisation overseeing the PICU.

4.2.16. The most prevalent and practicable model is one where the dedicated lead consultant is the same as the RC (within the meaning of the MHA 1983) for all the detained patients in a single PICU. 
4.2.17. There are variations on the most prevalent model described in 4.2.16. As an example, some PICUs have multiple RCs who manage the overall care of individual patients (or a small number of patients) in a single PICU. It is important to note that in such scenarios, the PICU should still have its own dedicated lead consultant, as described in 4.2.13-4.2.16 above.

4.2.18. The dedicated lead consultant for the PICU should have specific sessions or time-slots set out in the consultant job-plan to ensure consistent managerial and leadership input to the PICU and the wider forums that are associated with the PICU and its interfaces.

4.2.19. Other specialist professionals within the PICU team (e.g. psychologist, occupational therapist and pharmacist) should also exercise leadership in maintaining the quality, diversity and safety of the clinical approaches used within the PICU unit.

\subsection{Good practice guidance}

4.3.1. There should be an effective multidisciplinary approach within which all contributions from all members and all levels of the MDT are valued and carefully appraised. Consideration needs to be given to appropriate role definition concerning members of the MDT fulfilling interventions provided by the PICU. This improves team functioning and thus provision of care.

4.3.2. The available staff should be sufficient to meet the needs of the unit on a shift basis.

4.3.3. The PICU should be a dynamic clinical and learning environment through commitment to in-house and within-organisation training as well as external continuing professional development (see Chapter 17).

4.3.4. All members of the PICU core team should receive training in the methods for managing disturbed behaviour including restraint. This should also be the case for medical and non-nursing staff who may need to use the skills in an emergency and who require a clear understanding of the methods in use and their potential effects on patients. This can be best achieved by undertaking the training themselves in addition to the nursing staff.

4.3.5. Staff who are appropriately trained in the management of acute disturbance, including conflict resolution and physical interventions, should be available at all times.

4.3.6. A doctor should be available at all times to attend to an alert from staff members quickly when interventions for the management of disturbed or violent behaviour are required, consistent with NICE and other national guidance.

4.3.7. The unit should have access to interpreters, sign language and other communication media as appropriate.

4.3.8. Student placements also play an important role as students of all disciplines make a positive contribution to the care and treatment of patients.

4.3.9. There should be agreed processes for the consideration and resolution of conflict with the MDT. 


\section{Operations and clinical leadership}

\subsection{Rationale}

Management and leadership within a PICU are essential and symbiotic components in the establishment of the care ethos which the PICU holds. They are also fundamentally how this ethos is maintained within the clinical environment and therefore of great importance in the delivery of consistent and high quality care. Aspects of management and leadership which need to be specifically considered in relation to PICU are discussed in this chapter.

\subsection{Standard}

\section{Operational infrastructure}

5.2.1. The PICU unit manager and the lead consultant for the unit should be responsible for ensuring there is an adequate skill mix within the MDT for the PICU to deliver high quality care.

5.2.2. The PICU manager, along with the lead consultant, should be responsible for ensuring that there is:

- An operational policy for the unit;

- A clear mission statement including strategic aims, remit and goals of the unit and its treatment (which could be outlined within the operational policy);

- Clear systems and processes for the assessment, treatment and transfer of patients within the PICU.

5.2.3. The PICU manager and the lead consultant should be responsible for the implementation of clear systems and processes which underpin care within the unit.

5.2.4. The PICU should have a range of well-developed standard operating procedures (SOPs) which make up the PICU operational policy (see Chapter 6 \& Chapter 14).

5.2.5. It is each individual staff member's responsibility to familiarise themselves with and understand the SOPs. It is recommended that PICUs have the following SOPs in place:

- Admission

- Active treatment programmes covering the range of treatments available within the unit

- Preparing the unit for the arrival of an acutely behaviourally disturbed patient

- Handover and clinical review

- RT

- Unit mobile communication systems

- Interviewing an unpredictable or aggressive patient

- Searching for an AWOL patient

- Working alone

- Safety within the ward (e.g. necessary environmental security checks on a shift by shift basis)

- Entrance onto the PICU of support staff

- Transfer of patients post treatment. 
5.2.6. All SOPs should be based on current national guidance and local organisational policies. Where these conflict, this should be escalated by the PICU manager and national guidance should supersede.

\section{Operational capacity}

5.2.7. The clinical establishment of the PICU should not be used symbiotically with other services. For example, occasionally a place of safety (PoS) will be adjacent to or attached to the physical environment of the PICU. Staffing capacity for the operational running of the PoS should be in addition to the staffing establishment of the PICU and not interfere with the clinical capacity of the unit (see Appendix 2).

5.2.8. A shift management system should be in place to ensure that the PICU has acceptable levels of clinical expertise available at all times to treat and safely manage patients.

\section{Communication}

5.2.9. A member of staff should be allocated to each patient each shift. This should be communicated to the patient as soon as is practicable after shift changes if not before.

5.2.10. There should be clear processes in place to communicate with patients at all times, including during admission, treatment and transfer. An example of this, during treatment, could be a morning meeting with patients and staff on the early shift.

5.2.11. It is the responsibility of the allocated staff to ensure that prescribed care is delivered.

5.2.12. The shift co-ordinator holds the responsibility for shift management. This must be a suitably qualified and trained professional or professional in education. Where the shift co-ordinator is a junior member of staff, they should be closely supervised and supported by the senior professional on duty.

5.2.13. The handover of clinical and operational information is a key function in a PICU due to the potentially rapidly changing clinical presentations of patients. Therefore, a system should be in place which outlines how clinical hand-overs between all professionals are undertaken.

5.2.14. A formal handover between shift staff should be undertaken at shift changes. This should include details of all current patients in relation to:

- A summary of the events of at least the last three shifts

- Treatment plan

- Risk

- Vulnerability

- Suicide risk

- Violence and aggression

- Medication

- Progress

- Current assessment of needs

- MDT interventions

- Mental state examination (MSE) updates

- Other health and social care information (e.g. activity goals). 
5.2.15. A daily handover with other members of the MDT should be undertaken (especially medical staff).

5.2.16. An MDT clinical review should be undertaken for all patients within the PICU at least once a week.

5.2.17. Roles of the MDT should be well understood and articulated within the PICU. This will assist in role defining and prevent role blurring (see 4.3.1).

\subsection{Good practice guidance}

5.3.1. The PICU manager and lead consultant should be responsible for creating the conditions for high quality care, and for implementing processes for its delivery.

5.3.2. Ethos of care and good practice should be upheld and maintained by intrinsic system design based on current national evidence bases.

5.3.3. Leadership and role modelling is the responsibility of all staff members and is governed by the PICU manager and the lead consultant.

5.3.4. Safety within the PICU should be maintained by adherence to clear and standard procedures; this is the responsibility of all staff. 


\section{PICU care pathways}

\subsection{Rationale}

A clear and defined care pathway describing exactly how a person enters and leaves a PICU is required. Clear stages of care and an outline of journeys which involve psychiatric intensive care can serve to enhance patient and carer experience, clarify responsibility and add consistency to the quality of care. As a result, the patient receives the best possible care leading to optimal recovery in a timely fashion. This clear outline of care and expectations at each stage is referred to as a care pathway; in this case a specific PICU care pathway. The key principles underpinning care pathways are discussed in this chapter.

\subsection{Standard}

\section{Operational and clinical controls}

6.2.1. A care pathway can be seen as an application of process management thinking to the improvement of patient healthcare. An aim is to focus on the patient's overall journey, rather than the contribution of each speciality or caring function independently. More than just a guideline or a protocol, a care pathway is typically crystallised in the development of a consistent clinical approach to provide high quality care.

6.2.2. An operational policy is a document which adequately describes the objectives, principal functions and modes of operation of the entire unit. It refers to key organisational policies and local standard operating procedures (SOPs) specific to the PICU (see Chapter 5 \& Chapter 14).

6.2.3. SOPs describe an individual PICU's unique procedures and are not necessarily standard across units. They outline key processes which contribute to the quality, safety and consistency underpinning care delivery (see Chapter 14).

\section{Development and relationships of integrated governance standards}

6.2.4. All SOPs should be based on current national guidance and local organisational policies. Where these conflict, this should be escalated by the PICU manager and national guidance should supersede.

6.2.5. The introduction of all pathways governing care should be done in the spirit of good practice, and consistent and transparent service.

6.2.6. All pathways created, from overarching service pathways to specific clinical interventions, should incorporate up-to-date legal requirements, national guidance and best practice (e.g. Care Quality Commission, 2010).

6.2.7. The PICU should have an operational policy describing all care pathways, relating to care and processes within the PICU.

6.2.8. All processes of integral delivery and operations necessary to deliver high quality care should be outlined clearly within SOPs.

6.2.9. A PICU operational policy should be informed by and reference SOPs within the PICU; SOPs should also form the basis of the PICU pathway. An example can be seen in Figure 6.1. 


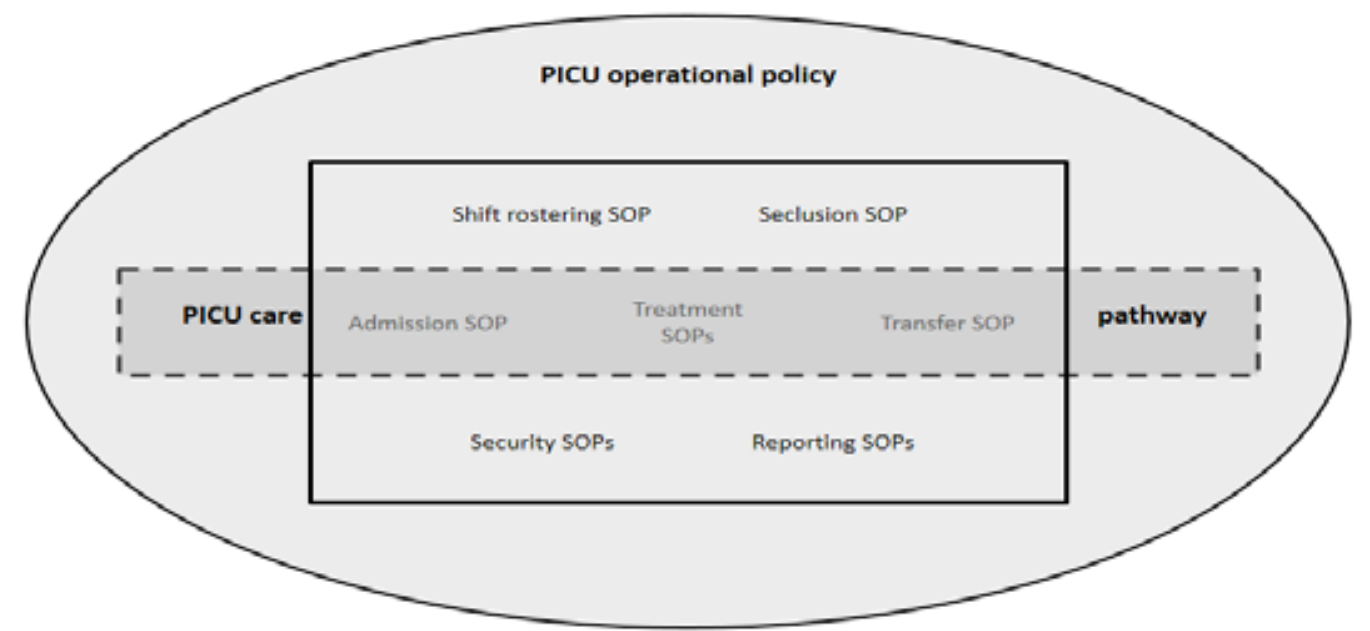

Figure 6.1. Example of the relationship between an operational policy, SOPs and a PICU care pathway

6.2.10. An integrated care pathway should be created in collaboration with the MDT giving due consideration to all aspects of care provided by the PICU.

6.2.11. The strategic importance of a PICU and its placement within an acute care pathway structure should be part of the PICU operational policy. An example can be seen in Figure 6.2.

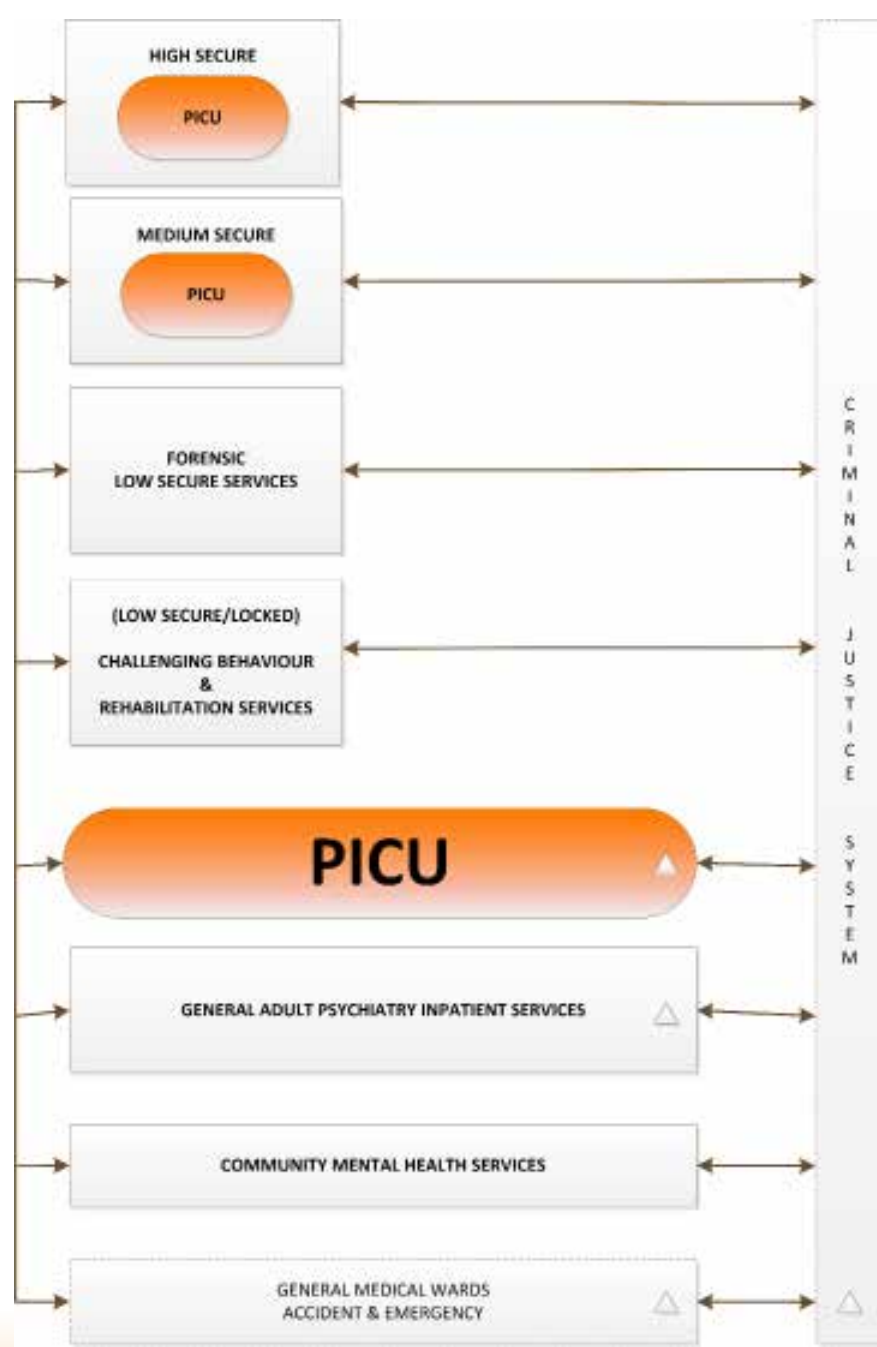

Figure 6.2. Example of the positioning of PICUs within the adult mental health service structure
Key:

Potential place of safety attached i.e. S136 suite Core service within mental health

Stakeholders who may be involved in care

6.2.12. In the development of a care pathway for PICU, consideration should be given to pre-admission, active treatment, transfer and post-transfer stages of care.

6.2.13. Where specialist pathways which fall outside of healthcare (e.g. the criminal justice system) are to be created and standardised, effort should be made to secure involvement from all parties in contact with the potential patients.

6.2.14. High level pathways, which include transfer in and out of the PICU, should include legal aspects and requirements (e.g. Section 19 transfer).

6.2.15. All care pathways should form part of the induction of clinicians and workers introduced within local acute pathways.

6.2.16. All referrals to the PICU should include an outline of all interventions already attempted to manage the patient in the host environment. This should be outlined in the purpose of admission. 
6.2.17. Interventional pathways created for specific treatments or conditions should be based on current national guidance, integrated governance and best practice.

\subsection{Good practice guidance}

6.3.1. All care pathways created should be evidenced based, legally compliant and underpinned by high quality and consistency.

6.3.2. All stages of care provision leading to PICU admission active treatment and follow-up should be considered in the development of comprehensive care pathways.

6.3.3. Psychiatric intensive care pathways should be adapted and standardised for local use.

6.3.4. The pathways designed for PICU should be as widely communicated as possible throughout the organisation, for maximum effect and understanding. 


\section{Physical environment}

\subsection{Rationale}

The physical environment of a PICU is one of its defining aspects. The design of any unit is key to its success and should maximise the primary functions of safety, therapy and security. However, it is acknowledged that this may be easier in a new building. Where physical changes are not possible (e.g. in some older buildings) considerable thought needs to be given to the processes and practices that are necessary to minimise any shortcomings.

\subsection{Standard}

7.2.1. The PICU should be on the ground floor.

7.2.2. If the PICU is part of a hospital, an entrance to the unit that does not necessitate travelling through the rest of the hospital should be provided.

7.2.3. From a clinical and operational perspective, recent evidence suggests that smaller PICUs tend to function better. As a maximum, no more than 14 beds are recommended.

7.2.4. Consideration should be given to the acoustics of the unit. Soft furnishings, wall coverings, ceiling type and carpet can all be useful in preventing echo and sound amplification. A balance may need to be drawn between risks associated with poor acoustics, unit feel and infection control preferences.

7.2.5. The unit's configuration and its fixtures and fittings such as window and door furniture, door closers and hinges, taps, shower heads and coat hooks should be anti-ligature, robust, able to withstand sustained attack, and meet national safety requirements. In general, all fixtures and fittings should be specified, manufactured, fitted and maintained to help prevent accidents, misuse, use as weapons or to aid self-harm. All safety and security measures, for example window restrictors, should be as discreet as possible.

7.2.6. Effective designs should be based on a risk assessment of the environment. For example, pipes, wires and heating elements should be hidden and inaccessible to patients.

7.2.7. Multiple corridors should be avoided in order to promote unobtrusive observation.

7.2.8. Wherever possible there should be clear lines of sight. This should also be possible around corners, by means of aligned windows or convex mirrors.

7.2.9. Corridors should be wide enough to allow three people to move abreast comfortably.

7.2.10. Ceiling height could be approximately $3 \mathrm{~m}$ in some areas, to give the feeling of space, and fitted with skylights that increase day light into the main part of the building.

7.2.11. There should be access to a quiet room for worship, meditation, reflection and counselling that is available to everyone who attends the unit. Every effort should be made to keep these resources away from the main patient areas and bedrooms.

7.2.12. The interior of the PICU should be smoke free. This should be achieved through the provision of a designated, safe outdoor smoking environment (although smoking cessation is encouraged, see 3.2.42). 
7.2.13. A family visiting room, which is warm, clean and well equipped, should be available outside the main body of the unit.

7.2.14. The dining area should be big enough to enable the patients to eat in comfort and to promote interaction and engagement between patients and staff.

7.2.15. In mixed gender units, the provision of gender specific areas such as bedrooms, sitting rooms, corridors, gardens bathrooms and toilets are required in national standards and guidance (NHS Executive, 2000).

\section{Garden}

7.2.16. There should be access to an enclosed secure garden, which should be of reasonable size to allow a feeling of some space.

7.2.17. All garden furniture should be immovable.

7.2.18. Gardens should only be accessed in the presence of PICU staff on a clear individual risk assessment basis.

7.2.19. At least a portion of the garden should be sheltered from the elements.

7.2.20. The garden fencing should:

- Be robust;

- Be constructed with anti-climb surface;

- In the case steel fences, consist of meshing which inhibits the easy passage of items;

- Include anti-climb measures;

- Include the provision of an emergency exit as an additional fire exit.

\section{Extra care area}

7.2.21. An extra care area (ECA) within the PICU can provide a quiet, low-stimulus space for patients experiencing high levels of arousal during periods of disturbed behaviour and can be used for de-escalation, patient support and management, and treatment in a bespoke space for high intensity intervention.

7.2.22. The ECA should provide for the daily living needs of a single patient for a limited period. It should include:

- De-escalation and/or seclusion room;

- Toilet and shower facilities;

- Sitting room with safe furnishings (i.e. robust furniture, which is recommended, or lightweight furniture which would not cause injury if thrown).

7.2.23. The ECA and any seclusion facility should be located in an area away from the main patient areas and bedrooms.

7.2.24. It should be possible to regulate both room temperature and ventilation in the ECA and in any seclusion facility.

7.2.25. The ECA should offer privacy from other patients, visitors and, when clinically appropriate, from staff with a maintained ability to observe. 
7.2.26. The ECA and any seclusion facility should be robust enough to withstand determined attack.

7.2.27. The ECA should not be included in the bed numbers for the unit.

\section{Seclusion}

7.2.28. Some PICUs operate without the use of seclusion. Seclusion is defined as a patient locked alone in a designated specialist space within the ECA, which must comply fully with standards 7.2.29-7.2.39.

7.2.29. Where seclusion is to be used, the room should comply with the standards detailed in the Mental Health Act Code of Practice (Department of Health, 2008).

7.2.30. The practice of using patient's bedrooms, or other designated rooms, as 'de facto' seclusion facilities must be avoided as this practice denies patients the protections established with the Mental Health Act Code of Practice (Department of Health, 2008).

7.2.31. PICUs which do not have seclusion facilities must ensure that local policies fully describe alternatives to seclusion and define how patients' safety, dignity, privacy, and health and well-being needs will be met.

7.2.32. The seclusion facility should be located in an area away from the main patient areas and bedrooms.

7.2.33. It should be possible to regulate both room temperature and ventilation in the seclusion facility.

7.2.34. The seclusion facility should be no less than $15 \mathrm{~m}^{2}$.

7.2.35. The seclusion room door should be of solid core design and robust; of no less than $55 \mathrm{~mm}$ in thickness and have a viewing panel with no blind spots into the seclusion room.

7.2.36. The seclusion room door must open outwards.

7.2.37. It should not be possible to reach any fixtures and fittings (including lighting), even from standing on the bed.

7.2.38. The bed within the seclusion room should not be moveable and should have padding which cannot be removed, is robust and easily cleaned.

7.2.39. Where seclusion is a maintained resource within a PICU, the room and practice must comply with current national guidance.

\section{Bedrooms}

7.2.40. Patient bedrooms should be single rooms and should include a staff call facility. The layouts of rooms, fixed furniture and equipment should ensure that the patient is not able to position themselves unseen in the room.

7.2.41. Anti-ligature wardrobe, drawers or shelves, a desk or work surface and a chair should be provided.

7.2.42. Consideration should be given to the installation of built in and protected televisions with provision for video and audio playback.

7.2.43. Bedrooms should not be overlooked and it should not be possible to approach the window closely from the outside to look into the room. 
7.2.44. Bedroom doors should have a vision panel to allow staff observation into the room; which can be controlled from the outside by staff only.

7.2.45. The extent of night time disturbance as a result of regular nursing observations should be considered. There are approaches to minimising the disturbance that can be caused by regular night time observation (see 7.2 .70 ).

7.2.46. It should be possible to open doors outwards in an emergency situation, so that it is more difficult to barricade the door and prevent others from entering.

7.2.47. Care is required to ensure that sound privacy is not compromised and that the emergency antibarricade system does not allow a view into the room.

7.2.48. Patients should be able to lock their rooms both when they are inside the room and on leaving it, to safeguard their property and to increase the feeling of safety, privacy and dignity. However, staff will need the facility to override the lock and the handles should be anti-ligature.

7.2.49. Electronic locks provide the opportunity for different levels of access for patients by means of an electronic fob which can be allocated individually.

\section{Main entrance}

7.2.50. An airlock design is required for the main entrance, comprising two doors set opposite to each other. Electronic locks should not allow one door to be opened until the other is closed. There should be an emergency override system in case there is a need to get a large number of staff in or out of the unit quickly.

7.2.51. The main entrance should be located away from the main clinical area so as diminish the extent to which it may represent a focal point for absconding attempts when the entrance is in use.

\section{Fire exits}

7.2.52. An agreed local procedure developed in conjunction with the building control officer or an approved inspector should be in place. The procedure should include measures that ensure the means of escape is controlled but not compromised. The use of key-operated call points may be considered.

\section{Doors}

7.2.53. Careful consideration should be given to the design of doors, frames and associated furniture to ensure that individually and collectively they minimise the opportunity for ligature risk and the means to barricade or to prevent the door from being opened.

7.2.54. All doors should be of solid core construction of at least $40 \mathrm{~mm}$ thickness, i.e. heavy duty design. (See 7.2.35 for additional guideline specific to seclusion room).

7.2.55. There should be as many doors as possible opening outwards.

7.2.56. Double (or wide single) doors should be installed in rooms such as the day room, dining room, activities room and other areas in which more than two patients gather. 
7.2.57. The handle on the outside of any door in an area accessible to patients should provide greater purchase than the handle on the inside of the same door.

7.2.58. The operating handle for the patient privacy lock on the inside of the bedroom door should be ligature-free.

\section{Windows}

7.2.59. Any unit design should aim for as much natural daylight as possible into the main clinical areas.

7.2.60. Glazing panels designed for use in secure environments should be fitted.

7.2.61. Windows should be constructed with a secure mesh where the passage of contraband is identified as a risk.

7.2.62. Specific security windows should be fitted with a security grill that allows the window to open enough to admit a significant breeze. For windows without a security grill, ventilation remains important and these should have a restricted opening of no more than $100 \mathrm{~mm}$.

\section{Locks and access control}

7.2.63. As many of the rooms as possible should be lockable.

7.2.64. Rooms such as the day and dining area should, for the majority of the time, remain open for free access by patients. There may be times however, when it may be necessary for these rooms to be temporarily restricted.

7.2.65. The kitchen area presents particular problems and should always be considered a potentially dangerous place.

7.2.66. Bathrooms and toilets should be lockable from the inside by means of a clutch or other similar arrangement; staff should be able to override these locks from the outside, with keys held by staff only.

7.2.67. Bedrooms and other rooms may also be locked from the inside with the same precautions as above, i.e. with an override system.

\section{Observation facilities}

7.2.68. As many clear lines of sight as possible should be available, avoiding numerous corners and corridors.

7.2.69. All doors (with the exception of those in bedrooms, bathrooms and toilets) should be fitted with a robust clear observation panel. This will enhance safety when moving around the unit by ensuring that the staff and patients can see the other side of the door.

7.2.70. There are products available that allow alternative methods of regular night time observation with the aim of minimising disturbance and maximising privacy (e.g. infra-red, breathing monitors). The value of such products should be considered in diminishing the disturbance caused by regular interval (usually a minimum of hourly) night time observation. 
7.2.71. Bathrooms and toilets may be fitted with a vision panel to allow staff observation into the room if required. Some means of obscuring the vision panel, which can be controlled by staff only, will be required. A number of solutions are available, including observation panels that change from white to clear or 'fish eye' lenses.

7.2.72. Switches with a dimmer, one located inside and the other outside the room should control bedroom lights. This will allow for night-time observation.

7.2.73. Where corridors meet, and in other areas without clear lines of sight, convex and other strategically placed mirrors can be fitted at ceiling level to allow views around corners.

\section{Closed circuit television (CCTV)}

7.2.74. Units should consider the potential value of CCTV as an area of innovation within a PICU in certain circumstances and areas in which it could be carefully deployed.

7.2.75. CCTV has proved useful by providing the following:

- Additional options for observation in difficult to supervise areas (e.g. gardens, smoking areas);

- A means of evidence, recording untoward incidents, potential offences or investigating allegations;

- An additional means by which staff can review the management of difficult situations;

- Up-to-date pictures of patients who may have absconded and are considered at risk.

7.2.76. The CCTV recording system should be easily assessable by designated staff with the appropriate training.

7.2.77. Any use of CCTV should be compliant with all data protection and other CCTV related legislation.

\section{Recreational, occupational therapy or other fittings}

7.2.78. An effective PICU design should give the provision of therapeutic activity an equal status to safety and security and should include:

- Activities room (containing board games, art and stereo equipment);

- Access to internet and social media (with appropriate safeguards in place);

- Day room (with a television and DVD player, or equivelent,);

- A room with physical exercise equipment.

7.2.79. Laundry facilities should be provided so that patient clothes can be washed in a timely manner.

\section{Furniture and fittings}

7.2.80. The unit environment should be made as comfortable as possible. For example, by using soft furnishings that strike an appropriate balance between safety, security and homeliness. The unit should make good use of pictures and other art to diminish the harshness of the environmental feel. The use of carpet in some areas is effective for improving unit feel and mitigating acoustic travel and amplification.

7.2.81. The unit should be fitted with a telephone to which the patients have free access (Department of Health, 2008). This could be on a portable trolley in case of persistent inappropriate use. 
7.2.82. All staff should carry a single device which allows them to raise an emergency alarm and to receive information regarding emergency alarms raised by others.

7.2.83. Wall mounted emergency buttons with audio-visual output are also a necessary fitting. These should be installed in addition to personal devices as they also offer protection for patients.

7.2.84. Emergency assistance call buttons should be placed in all rooms and at regular intervals along corridors.

7.2.85. The emergency system should be clearly understood by all staff (regular and non-regular, PICU and external). There should be a clearly documented and tested procedure for the use of alarms which includes guidance for patients on how to call for help using the alarms (see 8.2.6; 14.2.1.4).

7.2.86. Relevant assistive equipment such hoists, manual handling aids and handrails should be available to maximise independence in self-care needs.

\section{Communication systems}

7.2.87. There should be an established means of communication for the clinical team in clinical work outside the ward environment. For example during escorted leave, staff could use mobile phones or two-way radios.

\subsection{Good practice guidance}

\subsubsection{The PICU should provide:}

- Increased safety against aggressive, impulsive and unpredictable behaviour towards self and others;

- Prevention of absconding;

- Space for a range of therapeutic activities;

- Adequate space and facilities for a homely environment in which a patient can spend the majority of their day.

7.3.2. It is not possible to describe every detail of the ideal PICU physical environment. Further reading on specific PICU physical environment and design is recommended (see Dix \& Page, 2008). This design guidance is not overly prescriptive but is intended to provide the principles on which the PICU should be based. The design of PICU physical environments may also vary due to their differing functions. This standard should be read in conjunction with national guidance on single sex accommodation and current national healthcare building recommendations (Department of Health, 2010a).

7.3.3. Bedrooms should be en-suite. The design should allow for such facilities as television, radio, personal media equipment. Furniture and fittings within the bedroom should be built-in, where possible, including TV, video and audio players. Lockable personal storage facilities for clothing and possessions should be provided within each bedroom.

7.3.4. Effective care is best delivered when a range of semi-private and public spaces outside the private bedrooms are provided. These include social spaces, views to external areas, a quiet room and an interview room.

7.3.5. Innovations in technology should be considered where they provide a clear benefit to patients and providing that staff-patient relationships and relational security are not compromised. 


\section{Patient involvement}

\subsection{Rationale}

Patients rightly have high expectations of safe, quality care, delivered by efficient, caring and knowledgeable staff in a manner which engages them throughout their journey through the services. In order to deliver a genuinely patient-centred service, several processes should be created to enable patients to contribute to the design, delivery and monitoring of care. The aim is to promote an open, collaborative and transparent approach which actively supports patients being involved in their care from admission to beyond transfer.

\subsection{Standard}

8.2.1. A PICU should be able to demonstrate ongoing improvement and engagement activity with reference to recent publications (e.g. Cresswell et al. 2010; Lemmey et al. 2011; NICE, 2012; NHS Institute for Innovation and Improvement, 2012).

8.2.2. Each PICU should have a published statement outlining the opportunities for current and past patients to participate in their care and contribute to the unit operation and development.

8.2.3. The patient and carer (where appropriate) should be met on arrival, shown to an appropriate area and offered refreshments.

8.2.4. The patient should be introduced to a member of staff who will be their point of contact for the first few hours of admission.

8.2.5. The patient should be orientated to the unit environment, including toilets, as soon after admission as it is safe to do so.

8.2.6. On the day of their admission, or as soon as they are able to, the patient should be given and orientated to a written welcome pack or introductory booklet that contains a minimum of the following:

- A clear description of the aims of the unit;

- A clear description of the purpose of the unit;

- A simple description of the unit's philosophy, principles, and their rationale;

- A description of what is expected, including rights and responsibilities;

- A list of the unit team, including the name of the patient's consultant psychiatrist, PICU/ward doctor and key worker/primary nurse;

- The current programme and modes of available treatment;

- Visiting arrangements;

- Personal safety on the unit (see 7.2.83);

- Unit programme of activities;

- Unit facilities (e.g. garden, multi-faith room);

- Information on advocacy services;

- Information on how to raise a concern or complaint and who can support them in this;

- What practical items patients need in hospital and what should be brought in;

- Restricted and controlled items;

- Expectations for community living with other patients and staff within the unit. 
8.2.7. On the day of their admission, or as soon as they are able, detained patients should be given written information on their rights, including: rights to advocacy and second opinion, right to move hospital, right of access to interpreting services, professional roles and responsibilities, and the complaints procedures, in accordance with Section 132 of the MHA 1983. This should be done in a manner which explains the information and checks the patient's understanding.

8.2.8. The welcome pack should inform patients of resources available within the PICU and how to request anything additional which may be required but not specified. Patients should have access to resources that enable them to meet their individual self-care needs, including ethnic and gender specific requirements.

8.2.9. Each patient should be invited to meet a member of staff for one-to-one contact each waking shift and this should be documented. Time should be set aside specifically for this.

8.2.10. Recreational activities and interventions, including engaging in creative work, hobbies and special and social interests, should be available within the unit seven days a week and during evenings, particularly for patients without leave (see also 13.2.9).

\section{Involvement methods}

8.2.11. Storytelling; for helping patients to 'tell their story' in their own words can be useful, by promoting an understanding of the possibilities and connecting to other people's experiences.

8.2.12. Experience base design; for using patient (and staff) experience to design better healthcare approaches to improve quality. Listening to stakeholders' descriptions of their own experience of a service or pathway in order to understand differing perspectives of a journey.

8.2.13. Co-production; for an approach which recognises the resources and experiences that patients already have and delivers services with, rather than for, patients, their families and carers.

\section{Patient feedback processes}

8.2.14. In order to promote a positive, transparent culture that supports learning by engaging patients in processes which offer feedback mechanisms, the following areas should be considered, all to be guided by an agreed model of confidentiality:

\subsubsection{Clinical aspects:}

- Individual patient participation, contribution and involvement in their care and treatment;

- Patient feedback on their experience of safety and security within the unit;

- Patient engagement with and feedback on the design of a relevant social and therapeutic activities programme;

- Patient feedback on their experience of the unit's approach to managing high levels of acuity or disturbance or violent incidents;

- Patient feedback on their experience of developing their unique treatment or care plan and advanced statement or directive. 
8.2.14.2. Managerial aspects:

- Involvement with unit induction or orientation programmes;

- Patient representation on the operational or stakeholder forum, so as to promote users' views on:

- Therapeutic and recreational facilities

- Ward environment (e.g. bedrooms, ward furniture, décor)

- Planning of new facilities

- Operational policies and procedures

- Complaints

- Staff recruitment and training.

\subsubsection{Monitoring:}

- Involvement in the design, and auditing of, issues arising from clinical care (e.g. adverse incidents, near misses) as a learning process;

- Involvement in the design and auditing of real-time feedback mechanisms, patient and carer surveys;

- Involvement in the design of post discharge, or transfer, feedback mechanisms.

\subsection{Good practice guidance}

8.3.1 There are many patient national organisations with local branches (e.g. MIND, http://www.mind.org. uk/; NSUN, http://www.nsun.org.uk/) and many local patient organisations with specific interests. PICU staff and patients could benefit significantly from developing relationships with these groups to share developments, support training and pool resources. 


\section{Carer involvement}

\subsection{Rationale}

Carers for patients form an integral part of recovery and care, and provide a critical aspect in understanding presentation, acceleration of recovery and quality of care provision. As far as possible, carers should be involved as full partners in every appropriate aspect of the patient's care and treatment in order to maximise positive experiences and reduce stigma. This will also encourage active participation of patients and carers from the start of admission to discharge or transfer. All PICU environments should respond to carers concerns regarding treatment in a secure environment.

\subsection{Standard}

9.2.1. Staff are advised to refer to best practice and current guidance in engaging and considering carers roles and involvement, for example, The Triangle of Care self-assessment tool (Worthington \& Rooney, 2010) which emphasises the need for better local strategic involvement of carers and families in the care planning and treatment of people with mental ill-health. It also recommends better partnership working between patients and their carers, and organisations.

9.2.2. Carers should be involved at the beginning of care through the CPA process, especially during admission and transfer.

9.2.3. Sufficient basic demographic information should be checked and agreed with carers.

9.2.4. Carers and their essential role should be identified at first contact or as soon as possible afterwards (Worthington \& Rooney, 2010).

9.2.5. The PICU should provide written information about all aspects of the unit. This should be given to relevant carers within 24 hours of admission when possible.

9.2.6. All identified carers or relatives should be offered an interview with a member of staff within three days of the patient's admission to discuss their views about ongoing and future involvement in the patient's care.

9.2.7. Carers should be offered an explanation and information sheet about unit procedures, carer advocacy, welfare rights and mental health services.

9.2.8. All identified carers or next of kin should be informed within 24 hours of patient admission to, or transfer from, the PICU.

9.2.9. If any restriction to carer involvement applies then there should be a clear unit policy and carers should be notified accordingly.

9.2.10. Carers should be entitled, and feel able, to express their views to members of the MDT. This could be facilitated by regular interaction with the staff.

9.2.11. A crisis plan, especially for absconding, should include carers and any specific persons who may be at risk. 
9.2.12. Where requested, carer views on the care and treatment process (including transfer to and from the PICU) should be expressed in a face to face meeting with any one member of the unit team.

9.2.13. The PICU should have processes and an environment that provides safety, privacy and dignity during visits by carers.

9.2.14. The PICU should provide training for staff on meeting the needs of carers.

9.2.15. A carer support network or group should exist and be available to carers of patients admitted to the PICU.

9.2.16. A list of voluntary and statutory organisations that provide information and support for carers should be available on the PICU to ensure appropriate service provision, particularly for Black and Ethnic Minority (BME) groups.

9.2.17. Protocols for carers' assessment should be agreed with local CMHT and should form part of the of the CPA process.

9.2.18. All carers providing substantial care should have their needs assessed and a written care plan provided in line with the CPA.

9.2.19. All information leaflets about the PICU for patients and carers should be reviewed annually if possible, but at least once every three years (or additionally in line with any changes in organisational policies which affect practice) by a team including representatives of the local carers' support group.

9.2.20. Risk management plans should include risk to, and incorporate views of, carers (usually with the patient's consent).

\subsection{Good practice guidance}

9.3.1. Clinicians should be aware of the emotional impact on carers, the value of active carer participation and any potential difficulties that may result as part of this process.

9.3.2. All of the above should be guided by an agreed model of confidentiality compliant with The Data Protection Act 1998 (http://www.legislation.gov.uk/ukpga/1998/29/contents) and the patient's consent.

9.3.3. PICUs are advised to use nationally approved tools and models of carer engagement; for example, The Triangle of Care self-assessment tool (Worthington \& Rooney, 2010) and the NHS Friends and Family Test (Department of Health, 2012c). 


\section{Documentation}

\subsection{Rationale}

It is important that all PICUs have an information system that is adequate and effective. Inadequate systems and processes can compound any difficulties in communication. One of the main tools of communication is the documentation that is used to assess, plan and evaluate care as well as inform others.

\subsection{Standard}

10.2.1. A number of organisational policies and procedures will require specific documentation to be completed and kept for inspection (e.g. staff training records). Some processes within a PICU require specific documentation. Those listed below should be in place.

10.2.2. General documents:

- Operational policy of the unit (see 2.2.19; 6.2.2)

- Carers' pack (see Chapter 9)

- Welcome pack for patients \& staff (see 8.2.6)

- Security check

- Visitors' book

- Organisational operational policies

- Unit community meeting minutes.

10.2.3. Where possible, the PICU health record should be an electronic health record (EHR). The use of EHRs can aid the communication process and improve the quality of records management. The health record should contain the following:

10.2.3.1. Pre-admission documents:

- Referral form or documents

- Pre-admission assessment.

10.2.3.2. Admission documents:

- Demographics

- MHA 1983 section papers

- MHA 1983 rights forms (s132)

- Initial assessment (individual/MDT)

- Doctors' assessment

- Nurses' assessment

- Other MDT member specific assessments

- Medication record including medicines reconciliation

- Care plan

- Risk assessment/history

- Core physical health assessment

- Standardised and requisite payment by results (PbR) tool (where appropriate)

- CPA documentation

- Capacity assessment

- Property check and disclaimer. 
10.2.3.3. During admission documents:

- Incident forms

- Level of engagement/observation

- Section 17 form (where appropriate)

- Form T3/T2 (where appropriate)

- Primary/named nurse weekly summary

- Weekly MDT reviews' summary

- Management of aggression form (e.g. restraint, SCIP)

- Seclusion documentation

- Updated risk assessments and care plans

- Carers' assessment (where an existing assessment has not been completed).

10.2.3.4. Pre-transfer documents:

- CPA documentation

- Patient questionnaire outlining perception of treatment within the PICU (if appropriate)

- Treatment evaluation clinical tool (e.g. HONoS).

10.2.3.5. Post-transfer documents:

- Discharge summary from all disciplines within the MDT outlining problems, needs, progress and recommendations specific to each of the disciplines;

- Transfer sheet outlining current management plans and treatment details to facilitate smooth handover of care to receiving unit or team.

\subsection{Good practice guidance}

10.3.1. Documentation needs to be clear, concise and quickly accessible to all staff and regulators.

10.3.2. It is important that documentation on the PICU is in keeping with both local and national guidance documents.

10.3.3. Organisational policies that are especially relevant to the PICU are those regarding information management, confidentiality, prevention and management of violence and aggression, seclusion policy, MHA 1983 policies, RT and high dose medication policies.

10.3.4. Documentation on the PICU should be an aid for staff to ensure standards are met, including CPA standards.

10.3.5. Systems should be in place to enable third parties with a statutory right to access health records (e.g. regulatory commissioners) to access them without the need for the assistance of PICU staff, therefore ensuring staff are not directed away from patient care. 


\section{Ethnicity, culture and gender}

\subsection{Rationale}

All PICUs have the responsibility to ensure that all patients receive equality of treatment without prejudice or discrimination. This chapter discusses supporting mechanisms necessary within a PICU to set the conditions for objective and fair care delivery. In broad terms this would underpin wider national policies and local organisational guidelines in relation to equality. The principles of equality should apply to the whole PICU and include staff, patients, carers and others who have interfaces with the PICU.

\subsection{Standard}

11.2.1. The organisation in which the PICU operates should have clear policies relating to fair and equal treatment of all staff and patients based on national guidance, employment law and professional expectations.

11.2.2. The expectation should be that all PICU staff should have undertaken the mandatory equality and diversity training, and that senior managers on the PICU should have completed a higher level of training. It should be the responsibility of senior managers to engender a culture of embracing equality.

11.2.3. An outline of fair and equal treatment should be incorporated into the local PICU operational policy and incorporated into a welcome pack for patients as part of the overall philosophy of care. This can be demonstrated through equality of access to PICU provision and teams should reflect on the demographic data to create an appropriate environment within the PICU to provide a responsive service.

11.2.4. It is essential that all PICUs carry out local audits to look at the demographic profile of patients in the PICU setting and the use of the MHA 1983, and to compare this with national or regional benchmarks.

11.2.5. The PICU environment should, where possible, provide amenities and/or resources to accommodate the needs of individual patients (e.g. a multi-faith room, special dietary requirements). This is to ensure that the PICU is able to offer and respond to the needs of individual patients and respect any protected characteristic of individual patients.

11.2.6. The PICU should utilise the expertise and support of any local religious, cultural and pastoral services to respond to religious, cultural and spiritual needs. It is also essential to be aware of various ethnic religious festivals and to be sensitive to individual patients in enabling them to observe these festivities.

11.2.7. Specific patient needs should be established before admission or as close to admission as possible.

11.2.8. Assumptions about the patient based on their gender, culture, ethnicity or other personal characteristics should be avoided.

11.2.9. Where a PICU is mixed gender, the design, layout and procedures should be compliant with national standards for the provision of mixed gender accommodation (Department of Health, 2010a).

11.2.10. The PICU should have a clear policy about offering support to transgender patients, and patients undergoing gender reassignment, and at same time be respectful to the needs of other patients in the unit.

11.2.11. Any assessment tools used should, where possible, be sensitive to the patient's individual needs and should specifically directly involve those needs when they are directly affected by, or causal to, the acute presentation in consideration of holistic treatment approaches. 
11.2.12. Communication needs, and the need for interpreters, should be considered prior to admission, and arrangements for communication from and to the patient should be clear, to provide equal care. The communication needs of people with sensory impairment should also be considered, and appropriate support should be in place for these patients during their inpatient stay.

11.2.13. In areas with a predominance of a particular ethnic group, the PICU should consider appointing bilingual support workers.

11.2.14. The PICU should have appropriate mechanisms to engage carers and be sensitive to the needs of carers from diverse groups.

11.2.15. All ward information should be available in a variety of languages and media suitable to facilitate good communication and understanding between patient, carers and health professionals.

11.2.16. Care plans should incorporate aspects of specific cultural, ethnic, gender specific care provision necessary to facilitate needs where possible. The care plans should be recovery-focused and sensitive to the diverse aspects and protected characteristics of the individual.

11.2.17. Where specialist treatments are identified within assessment, the PICU should attempt to address these needs with specialist providers, or provide sign-posting to appropriate post PICU interventions for the patient and carers (see also 3.2.3).

\subsection{Good practice guidance}

11.3.1. All organisational and PICU policies within organisations which facilitate PICU care should be aligned to treating in the most holistic way possible relating to age, ethnicity, culture, individual need and respecting any protected characteristic (see 20.2.4 - 20.2.9 for further guidance on female-only PICUs).

11.3.2. Within the PICU environment, whilst risk assessed, all aspects of individual need pertaining to treatment should be addressed as fully as possible without prejudice or discrimination.

11.3.3. The resources within a PICU should be set up to cater for the needs of the local demography as much as possible.

11.3.4. National resources (e.g. Care Quality Commission, 2011) should be utilised to provide a framework for the MDT to develop best practice and reduce inequality. 


\section{Supervision}

\subsection{Rationale}

Staff supervision is widely accepted as an essential prerequisite for high quality care. The provision of supervision for healthcare staff is a regulatory requirement in the UK. Teams should have well defined and robust systems of clinical supervision in addition to managerial supervision. The provision of clinical supervision to all members of an MDT is recognised as being challenging.

\subsection{Standard}

12.2.1. Staff supervision should occur at a minimum of once every four weeks or more frequently as per professional body guidance.

12.2.2. Supervision should be recorded and each PICU should have a clear system of monitoring and auditing supervision. This should be reviewed every six months.

12.2.3. The supervision process should be explained to any new member of staff during induction.

12.2.4. The requirement to participate in supervision should be included in the job descriptions for all members of the team.

12.2.5. All PICUs should have clear clinical supervision guidelines which incorporate supervision contracts between supervisor and supervisee to cover:

- Learning/training objectives

- Resolution of conflict (arbitrator identified)

- Roles and responsibilities

- Practicalities (e.g. location)

- Boundaries (e.g. time and agreed agenda)

- Documentation to be used

- Confidentiality (adherence to professional code of conduct and organisational policy)

- Actions in event of non-attendance or cancellation

- Frequency \& duration.

12.2.6. All PICUs should have a list of appropriate clinical supervisors.

12.2.7. Non-regulated health and social care support workers should receive supervision from a registered practitioner.

12.2.8. Emergency ad hoc supervision and other support measures should be available in times of crisis and following untoward incidents.

12.2.9. All students and doctors in training should have access to clinical supervision. 
12.2.10. Where managerial and clinical supervision is combined, supervision should adhere to a specific structure (relevant to the appropriate professional body) and also address both clinical and managerial factors including:

- Monitoring (including patients' concerns)

- Caseload supervision

- Development (core skills \& training for staff)

- Self-development

- Support in stressful situations

- Action plans (shared agreement of actions)

- Safeguarding adults.

12.2.11. All staff should participate in a defined annual appraisal, where progress is measured against agreed standards and actions are identified to develop the staff member.

\subsection{Good practice guidance}

12.3.1. A comfortable and private room away from the workplace, free from interruption, should be identified for supervision.

12.3.2. A relevant evidence-based model for clinical supervision should be identified and implemented within the unit.

12.3.3. Staff should be able to choose their clinical supervisor.

12.3.4. The effectiveness of clinical supervision should be periodically assessed. Possible effectiveness evaluation could be completed through the following:

- Staff surveys;

- Measurement of occurrences of issues highlighted by the clinical leadership and addressed by the MDT though supervision;

- Supervision audit and review of completeness, compliance with organisational standards for appraisal or use of evidence-based tools such as the Gallup 12 Questions Survey (Wagner \& Harter, 2006). 


\section{Liaison with other agencies}

\subsection{Rationale}

PICUs should ensure that mechanisms are in place for immediate access to agencies or services in order to provide rapid assessment, to ensure that admission to the PICU is timely and appropriate, to formulate and monitor management plans and plan prompt discharge or transfer from the PICU. Continuity and consistency of care, and establishing reliable and empathetic relationships with healthcare professionals, are key to patients receiving appropriate, effective and high-quality care. Relevant information should be shared between professionals to support this and to ensure effective coordination.

\subsection{Standard}

13.2.1. Each PICU should have a clearly identified and documented contact or link person in each agency involved with the patient.

13.2.2. Links should be made with agencies under six broad categories:

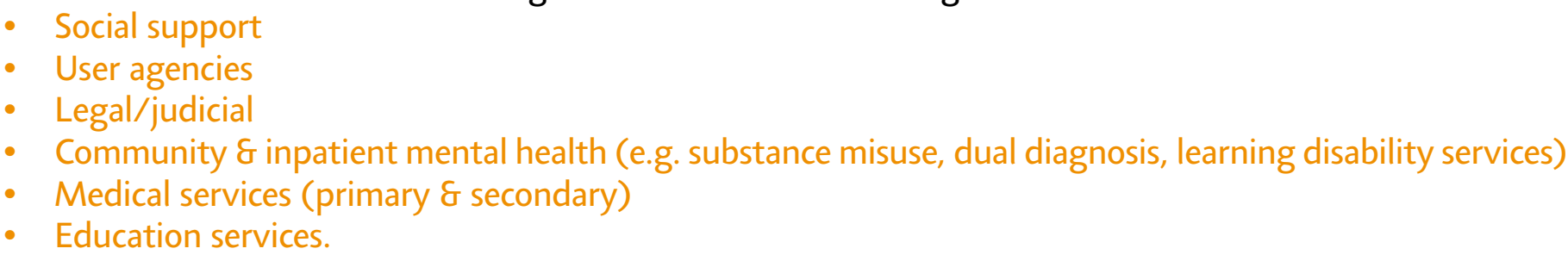

13.2.3. The PICU should disseminate information about the unit to all relevant agencies.

13.2.4. The PICU, in liaison with the relevant agencies, should develop clear protocols about shared level of involvement.

13.2.5. Assessment of referrals to the PICU and communication of the outcome of assessment should take place before admission to PICU is agreed.

13.2.6. During admission to the PICU, weekly contact should take place between the PICU and the unit from which the patient has been admitted (and is most likely to be transferred, discharged or returned to).

13.2.7. In accordance with individual care plans, all possible links to other agencies identified should be passed on to the relevant mental health team to ensure continuity of care.

13.2.8. Planning for transfer from PICU should be agreed and clearly communicated between the PICU team, accepting team and other teams involved in the patient's care, such as an intensive team, early intervention team, specialist teams and other agencies.

13.2.9. Patients should have information on any other agencies involved in their care, be made aware of who to contact, how to contact and when to make contact about their ongoing healthcare needs. The care plan should reflect this.

\subsection{Good practice guidance}

13.3.1. It is the responsibility of all PICUs to develop effective networks with other agencies:

- To maximise the quality of the care package available to individuals;

- To maximise engagement and therefore improve quality of life and future well-being of individuals;

- To maximise the available resources and empower the patient to choose what is best suited to their needs;

To reduce stigma attached to treatment in a secure environment. 


\section{Policies and procedures}

\subsection{Rationale}

A policy is a statement of an organisation's position and philosophy on key issues, setting out a framework and rules of practice within which staff operate. Operational policies and procedures essential to the operation of a service are required to ensure there is an overarching governance structure in place to maximise the primary functions of safety, therapy and security.

\subsection{Standard}

14.2.1. It is recommended that the following policies should be in place:

14.2.1.1. An introduction to, or description of, the service including the aims of the service.

14.2.1.2. Procedures governing access to and transfer from the service including:

- Referral processes (e.g. response times, eligibility, exclusion criteria);

- Interfaces with health agencies (e.g. mental health and acute medical services);

- Interfaces with criminal justice system (e.g. police, courts, prisons, CPS, MAPPA);

- Interfaces with others (e.g. social services, housing).

14.2.1.3. Procedures governing risk assessment and management including:

- Risk assessment and management from admission to transfer;

- Prevention and management of violence and aggression including de-escalation, de-stimulation and restraint;

- Risk assessment and management of untoward incidents (e.g. suicide, self-harm, violence \& aggression);

- Building security checks.

14.2.1.4. Procedures governing security and personal safety (see Chapter 18) including:

- Control over the physical environment (see Chapter 7);

- Testing of safety enhancing systems on a PICU (e.g. personal alarms testing, fire evacuation);

- A clearly outlined role for a security nurse which is maintained on every shift;

- A clearly identified physical intervention capability (i.e. the ability to safely restrain a patient for their own safety or the safety of others) on every shift.

14.2.1.5. Procedures governing treatment including:

- Behavioural approaches to treatment;

- Therapeutic activities programme including MDT roles;

- Medication including use of high dose medication and RT (in compliance with current national guidance and reviewed regularly);

- Medical emergencies including resuscitation;

- MDT reviews and CPA review meetings.

14.2.1.6. Procedures governing induction and orientation including:

- Patient induction and orientation (see 8.2.3-8.2.7);

- Information policy and induction for carers (see 9.2.5-9.2.7);

- Visiting times and procedures;

- Children visiting arrangements on or off unit;

- Staff induction and orientation (see 16.2.4-16.2.6) 
14.2.1.7. Procedures governing interventions consisting of a restriction of movement or deprivation of liberty including:

- Seclusion;

- Special or extra care areas;

- Escorted and unescorted leave considering concepts of 'custody' during leave;

- Missing person or absent without leave policy;

- Observation and supervision of patients;

- Search policy including patients, rooms and visitors;

- Management of substance misuse including use of and supply to others.

\subsubsection{Procedures governing access to the physical environment including:}

- Provision and return of keys, alarms, swipe cards, RFID tags;

- Reception, induction and airlock protocol;

- Clear list of, and information on, items considered contraband;

- Building access (e.g. deliveries, ancillary staff, emergency access);

- Access to toilets, bathrooms, bedrooms;

- Use of secure garden;

- Use of extra unit facilities on and off site;

- Equipment storage.

\subsubsection{Procedures governing legal and other issues including:}

- Provision of reports for manager's hearings and MHA 1983 review tribunals;

- Information on mental health legislation or where to access such information;

- Issues of capacity and consent;

- Issues of confidentiality and data protection (see 2.2.18);

- Patient access to social media during treatment;

- Sharing information with the criminal justice system;

- Police interview or court attendance procedures;

- Taking body fluids for medical and legal reasons;

- Security and monitoring of patients' property and money;

- Harassment, intimidation, abuse or violence toward patients, staff or visitors.

14.2.1.10. Procedures governing human resources and staff development including:

- Bank and agency staff (e.g. booking, induction and information giving);

- New regular staff induction, mentoring and preceptorship;

- Mandatory training including skills updates;

- Staff support systems including regular supervision and exceptional support (e.g. following an untoward incident);

- Responsibilities ensuring the progress of personal development plans;

- Clinical supervision;

- Managerial supervision.

14.2.1.11. Procedures governing equality, diversity and anti-discriminatory practices including (see Chapter 11):

- Equal opportunities for staff and patients;

- Training in equality and diversity;

- Improving cultural competence;

- Production and use of translation services for written material and interpreting services for patients who do not have English as a first language;

- Provision of single gender areas;

- Responding to patients' spiritual needs and preferences. 
14.2.2. All policies should be updated in line with national and organisational guidance, and subject to an equality impact assessment.

\subsection{Good practice guidance}

14.3.1. A number of policies may be specific to a particular department or unit.

14.3.2. In the absence of an organisational policy regarding a specific area of practice, policy development groups should include staff required to implement those policies and staff those policies impact upon.

14.3.3. When developing specific policies for the environment, consideration should be given to:

- Policy statement

- Rationale

- Evidence

- Competencies required

- Implementation

- Monitoring

- Evaluation and review methods. 


\section{Clinical audit and monitoring}

\subsection{Rationale}

Clinical audit is at the heart of clinical governance (NICE, 2002) and is an essential tool in raising the quality of care through:

- Assessing the quality of practice against agreed standards;

- Highlighting areas of concern regarding the quality and cost-effectiveness of patient care;

- Improving practice through informed feedback;

- Being an integral part of service culture in order to monitor service responsiveness to the various aspects of patient care.

\subsection{Standard}

15.2.1. All mandatory and statutory audits (e.g. environmental audits, record keeping, audits on the use of RT) should be completed at the required frequency and if results show less than satisfactory practice, action plans should be put into place and enacted to rectify this.

15.2.2. The PICU should participate fully in relevant organisation-wide audits of practice.

15.2.3. All audits which involve patients' personal information should be subject to relevant governance, most recent national guidance and appropriate ethical approval as necessary.

15.2.4. The MDT member responsible for overseeing each clinical audit should have a good understanding of the function, methods and application of clinical audit.

15.2.5. The MDT members responsible for audit and monitoring should adhere to regulations of the Data Protection Act 1998 (http://www.legislation.gov.uk/ukpga/1998/29/contents) and supplementary documents.

15.2.6. Patients and carers should be involved in the process of clinical audit as far as possible.

15.2.7. Service responses to the ethnic, cultural and gender needs of patients should be audited regularly.

15.2.8. Results of clinical audit should be disseminated to all stakeholders with recommendations regarding future practice; action plans should be developed and monitored to address any substandard practice.

15.2.9. Audits should be carried out at various different levels of operational delivery to ensure that conditions and delivery meet the required national and local standards. Examples of different types of audit follow.

15.2.10. All PICUs should have clear processes in place to implement action plans in response to audit findings; for example, to address areas that need improvement following national or local patient surveys and feedback (Care Quality Commission, 2011).

\section{Extra-organisational}

15.2.11. The PICU should carry out regular mock inspections based on national mandatory standards (e.g. CQC standards). 
15.2.12. The PICU environment should be audited, based on national frameworks for inpatient services such as Patient Environment Action Team (PEAT) or The Patient Lead Assessment of the Care Environment (PLACE).

15.2.13. The PICU should comply with national performance standards in terms of information provision (e.g. average length of stay and re-admission rates).

\section{Organisational}

15.2.14. The PICU should, along with other services, be involved in a regular audit of compliance of care under the CPA.

15.2.15. A regular audit of all clinical records for compliance with set quality standards should be undertaken at least quarterly.

15.2.16. The PICU should have annual infection control audits.

15.2.17. There should be at least an annual health and safety visit to the ward, including an executive board member of the organisation.

15.2.18. The PICU should have a mechanism for recording satisfaction levels of patients and carers; for example, the national Friends and Family Test (Department of Health, 2012c).

\section{Service level}

15.2.19. The PICU should audit:

- Physical monitoring of patients (including on admission and during active treatment);

- Compliance of treatment and adherence to MHA 1983 legislation;

- The presence, relevance and overall quality of care planning;

- Supervision rates of all staff working within the unit;

- Training compliance with both mandatory and statutory training;

- Maintenance and administration of medication.

\section{Unit level}

15.2.20. The PICU should be provided with reports based on incidents of restraint, complaints, medication errors, incident rates, verbal and physical abuse, and praise.

15.2.21. The PICU should monitor overall incidences of restraint, successful de-escalation (or attempts to de-escalate), RT, seclusion and length of any seclusions.

15.2.22. The PICU should undertake audits of controlled drugs, and their stock, within the unit.

\section{Patient level}

15.2.23. The PICU should monitor the frequency of one-to-one care for the patient with their named nurse or key worker. 
15.2.24. Essential high risk activities should be accurately documented, audited and reviewed to ensure good clinical practice and positive learning. These activities could include:

- Restraint

- Seclusion and other restrictive practices

- RT

- Use of high doses of psychotropic medication

- Presence and use of equipment and medicines for the treatment of medical emergencies

- Adverse incidents and 'near misses'.

\subsection{Good practice guidance}

15.3.1. Standards in this document should be selected for auditing.

15.3.2. As a key tool in delivering clinical governance, clinical audit should be part of routine practice within a PICU. Higher levels of risk, loss of liberty and legal status of patients suggest a greater need for clinical audit and monitoring than usual, with particular attention paid to areas such as: observation, seclusion, restraint and RT.

15.3.3. For clinical audit to be successful, the MDT needs to give full co-operation to the implementation of the audit process including incident reporting, review of incidents and 'near misses' and generation of good practice principles. This process should be based on a reflective learning approach to practice so that positive learning can occur from mistakes.

15.3.4. Units are expected to participate in relevant national audits and surveys of practice in order to benchmark their services, to contribute to the wider dataset and understanding of current practice and to help shape future service delivery. 


\section{Staff training}

\subsection{Rationale}

In the highly demanding and potentially stressful work environment of a PICU, it is essential that staff are well-trained. In the current climate of evidence-based practice, it is also important that clinicians are up-todate with the knowledge and skills pertaining to their specialist area, in addition to maintaining the values and attitudes required to provide high quality care.

\subsection{Standard}

16.2.1. There should be a organisation-wide strategy in place to ensure that training is available, with a specific training matrix to address needs identified for psychiatric intensive care workers.

16.2.2. The PICU should be considered to be a high stress environment and provision should be made for a staff away day at least annually.

16.2.3. Access to training should be facilitated and there should be arrangements for staff cover to allow others to attend training.

16.2.4. Before being asked to carry out any clinical work, all staff should receive mandatory training in fire safety, manual handling and basic life support.

16.2.5. Staff should complete a unit-based induction programme which ensures basic understanding of core skills. Where additional training is identified this should be delivered and prioritised according to safety and quality impact, and patient need. This training should be delivered, and the new staff member able to demonstrate those competencies appropriate to their job or role, within six months of joining the team.

16.2.6. All staff should complete a security induction programme prior to being issued with secure keys, pass cards or fobs.

16.2.7. All new staff should be allocated a mentor who oversees their induction.

16.2.8. All staff should attend identified statutory and mandatory training, including risk assessment and safeguarding, and six other practice-associated days per year.

16.2.9. Organisations should provide access to training that equips healthcare professionals with the competencies required to deliver the psychological therapy interventions recommended in these standards.

16.2.10. The unit budget should be sufficient to enable staff to attend the required training.

16.2.11. All staff should additionally participate in Continuing Professional Development (CPD) (see Chapter 17).

\section{Clinical staff}

16.2.12. A significant proportion of the MDT should be able to provide low intensity psychological interventions in line with NICE guidance.

16.2.13. Continuing training and refresher courses (e.g. interpersonal skills) should be available for all staff.

16.2.14. Managerial supervision is the vehicle to ensure that these core skills can be demonstrated. 
16.2.15. Regular training in addressing inequality issues and developing cultural competence should be available to all staff. This should lead to understanding and sensitivity in meeting patient's ethnic, cultural and gender needs (see Chapter 11).

16.2.16. Staff who undertake assessment and care planning should have received training in:

- Risk assessment, management and risk-taking

- De-escalation techniques

- RT

- Management of violence and aggression

- Use of restraint techniques

- Assessing mental capacity

- Self-harm and suicide, awareness and prevention techniques

- CPA including transfer/discharge planning

- Assessing carers' needs

- Diversity awareness

- Safeguarding children and vulnerable adults.

16.2.17. All practitioners who administer medications should undertake a competency assessment annually; records of this assessment should be available on the unit.

16.2.18. All prescribers should undertake an annual assessment of competency in the prescribing of medications; records of this assessment should be available on the unit.

16.2.19. All staff based on the unit should be trained in the management of disturbed behaviour.

16.2.20. All regular and temporary staff should be trained in breakaway techniques and conflict resolution including therapeutic management of violence and aggression.

16.2.21. All education and training in the safe and therapeutic management of aggression and violence should be based on current national guidance and contain significant elements on prevention and de-escalation.

\section{Multidisciplinary training}

16.2.22. There should be an investment in the development of managerial and clinical leadership competencies of unit managers and team leader grade nurses. This leadership development programme should be in line with current national healthcare leadership frameworks.

16.2.23. The appropriate clinical lead should be accountable for providing opportunities for new staff to acquire and/or demonstrate the appropriate competencies.

\section{Medical staff}

16.2.24. Training should be provided in prescription of high dose medications and augmentations; this includes specific training for the prescription of RT.

16.2.25. Training should be provided in undertaking physical examinations of patients.

16.2.26. In-depth training should be provided in relation to the MHA 1983 with specific emphasis on Part III.

16.2.27. Training for Section 12 approval under the MHA 1983 should be a requirement of all practising consultant psychiatrists within the PICU. 


\subsection{Good practice guidance}

16.3.1. A training programme for organisation and employee requirements should be available to all staff who work in a PICU.

16.3.2. A specific training programme to cater for the specific clinical, interventional and skill development necessary to develop within a PICU setting should be available and tailored to the MDT.

16.3.3. The training programme should, where possible, provide the necessary skills to meet requirements of national guidance for clinical interventions.

16.3.4. The training programme should, where possible, provide training for leaders to develop the clinical and work environment of the PICU over time.

16.3.5. The training programme should provide the MDT with the overall ability to deal with most daily operating requirements of a PICU.

16.3.6. Appendix 1 provides further guidance on staff training. 


\section{Continuing professional development}

\subsection{Rationale}

Continuing professional development (CPD) should meet the needs of patients and help organisations to deliver their objectives for improving healthcare, as well as assisting individuals to expand and fulfil their potential. Effective CPD relies on shared understanding between units, staff, regulatory bodies, and learning providers. Building a learning environment is essential in every PICU, to promote lifelong learning and support excellence in clinical care.

\subsection{Standard}

17.2.1. All registered practitioners are required to undertake CPD as a requirement of registration and revalidation. Non-registered staff should also undertake CPD.

17.2.2. A programme of individual CPD should be detailed in an agreed framework, such as the appraisal process.

17.2.3. All staff training should be underpinned by a clear philosophy on the provision of care within the PICU and address the specific challenges that working in a PICU presents.

17.2.4. To ensure the provision of the required interventions in a PICU, a training needs analysis should be completed annually to inform CPD planning.

17.2.5. Non-registered members of the PICU team frequently spend significant periods of time with patients. These staff should be supported to develop skills in listening, conversation, observation and engagement as well as primary prevention and de-escalation.

17.2.6. Team members should ensure that their CPD has contributed to the quality of their practice and service delivery and to ensure that their CPD benefits the patient.

17.2.7. The following core competencies should form the basis of an individual's training and development plan:

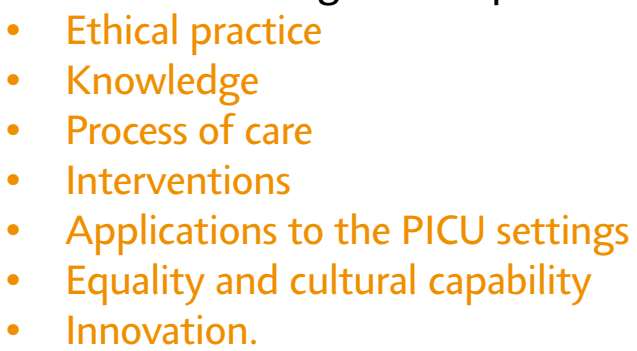

17.2.8. The unit budget should be sufficient to enable staff to participate in CPD minimum standards.

\subsection{Good practice guidance}

17.3.1. CPD is a continuous process of learning. It should focus on improving the skills and knowledge of staff so as to ensure that care provision is of a high quality and that admission to the PICU is a beneficial experience for patients. To achieve this, staff should aim to take up training and development opportunities provided over and above those legally required for each post. 
17.3.2. The PICU should be able to demonstrate that CPD activities are a mixture of learning activities relevant to current or future practice that support staff to maintain a focus on quality improvement.

17.3.3. Within high functioning teams, responsibility for improvement should be devolved to frontline staff, and acts of leadership can come from anyone within a healthcare team. All team members can participate in leadership development which will ensure that the whole workforce can have the leadership knowledge, skills and behaviours needed to improve health and care. 


\section{Security and risk assessment}

\subsection{Rationale}

The provision of a degree of security is one of the primary functions of the PICU. This needs to be counterbalanced with the need for a therapeutic clinical environment. The model of security should be integrated with dynamic risk assessment and management processes. A clear model of security and patient focused risk management leads to a safer therapeutic service, where patients experience a more balanced holistic approach.

\subsection{Standard}

\section{PICU security matrix}

18.2.1. The PICU should normally operate four dimensions of a security matrix, which are interdependent and should be managed jointly:

- Physical security

18.2.2. Physical security includes all aspects of PICU unit design including the use of equipment and technology.

18.2.3. Procedural security is the system by which safety and security is maintained and involves the effective application of policies, practice guidelines and clinical/operational protocols, allowing staff to engage in safe practice with confidence and consistency. Chapter 10 and Chapter 14 provide guidance on the documentation, policies and procedures required in the general adult psychiatric PICU.

18.2.4. Relational security is the most important of all of the security domains as increases in relational security diminish the prominence of the other security domains and the need for restrictive interventions.

Relational security is about understanding the patient within the clinical environment, and translating that understanding into high quality care.

18.2.5. The treatment model is part of the security matrix, and this is one of the defining features of psychiatric intensive care.

\section{Additional considerations for security}

18.2.6. Adult PICUs which are managing patients requiring physical security akin to that of a low secure unit should have regard for low secure standards. Chapter 7 provides guidance on the physical environment characteristics of the general adult psychiatric PICU.

18.2.7. PICUs in medium secure and high secure settings should comply with the appropriate corresponding physical security specification.

18.2.8. PICUs in medium secure and high secure settings should have a level of procedural security which is appropriate for the associated security specification. 
18.2.9. Good relational security is associated with high staff-to-patient ratios, continuity within the team, effective multidisciplinary working, a well-trained clinical team with a suitable skill-set, an approach based on individualised care for the patient, and a commitment to a therapeutic programme in the PICU. When thinking about relational security, which is applicable to all mental health services, the PICU should use approved national models, for example, See, Think, Act, a model developed by the Department of Health (Department of Health, 2010b).

18.2.10. The significance and impact of relational security measures are applicable to all PICUs, but at higher levels of security are of a more complex nature and of a higher intensity.

18.2.11. The model of intensive and dynamic clinical risk assessment and management should be considered on an equal footing with other security dimensions.

\section{Risk assessment and management}

18.2.12. On admission, there should be an immediate to short-term risk assessment of the patient. In the past, such risk assessment has been undertaken in a somewhat unstructured manner.

18.2.13. A current area of innovation and development in PICU research is the use of structured clinical assessment tools focusing on the short-term prediction of behavioural disturbance. The superiority of such tools over standard structured clinical judgement remains unproven as yet.

18.2.14. In a PICU, it is strongly recommended that the immediate to short-term risk assessment should be documented shortly after admission, preferably in a structured format that is replicated for all patients, but has the latitude to apply to unusual risk scenarios.

18.2.15. In the UK, using the CPA is recommended; the structure of which incorporates a general risk assessment and management scheme.

18.2.16. The immediate to short-term risk assessment of the PICU patient should include the following:

- Capacity status and associated consent to treatment;

- Nature and severity of risk indicator leading to PICU transfer;

- Risks to self or others of a physical, psychological or sexual nature;

- Risks of self-neglect or other vulnerability within the PICU;

- Risks associated with physical health disorder;

- Risks associated with psychopathological signs;

- Risks of alcohol or substance use (both licit and illicit);

- Risks associated with absconding;

- Adult safeguarding, children safeguarding and wider public protection;

- Risks associated with criminal intent or behaviour;

- Areas of possible risk that need further exploration.

18.2.17. As the PICU admission progresses, the (baseline) immediate to short-term risk assessment should be updated regularly by the MDT. These updates should be informed by further historical clinical and risk information gained by the team, set in the context of the patient's ongoing clinical progression and response to treatment. 
18.2.18. The PICU will often require more specialised clinical risk assessment tools. For example, where patients present with risks associated with violence, sexual violence, suicide, psychopathy, stalking, firesetting, malingering and personality disorder. Such risk assessment tools should be used by clinicians with the appropriate expertise, training and clinical experience.

18.2.19. Actuarial approaches to risk assessment are an invaluable tool in PICUs, but they should be used with caution as patients in the PICU often present with rapid behavioural changes due to the acute phase of a serious mental disorder.

18.2.20. Risk assessment and risk management should happen at the same time in a PICU. Risk assessment and risk management in the PICU should form part of the general system of regular MDT review of the patient's clinical care and treatment.

18.2.21. In spite of the critical nature of the risks managed in the PICU, clinicians should have regard for all of the following when considering risk management plans in the PICU:

- The patient's capacity to consent to information sharing;

- The patient's right to confidentiality;

- The patient's right to be involved in the risk management planning;

- The impact of any advance directives made by the patient;

- The inclusion of the carer perspective in risk management planning;

- The legislative framework regarding information sharing with victims;

- Any lawful impediments placed upon the patient;

- The question of whether increased containment may lead to higher risk.

18.2.22. The balance between protective restriction and individual autonomy should be struck to allow for positive risk taking with reasonable safeguards.

\subsection{Good practice guidance}

18.3.1. The PICU should operate four interdependent dimensions of a security matrix: physical security; procedural security; relational security; and treatment model security.

18.3.2. On admission to the PICU, there should be an immediate to short-term risk assessment of the patient.

18.3.3. The risk assessment should be updated regularly by the MDT, as more detail emerges of the patient's historical and current risk profile. 


\section{PICU support infrastructure}

\subsection{Rationale}

Many staff external to the unit are essential for good care delivery and treatment but may not be aware of safety and security issues within the PICU environment. A system needs to be in place that informs nonregular staff and others of procedures that they need to adhere to while in the PICU. External staff may be defined as anyone seeking entrance to the PICU area who is neither a patient of the PICU nor a member of the MDT employed within the hospital.

\subsection{Standard}

19.2.1. A local SOP should be in place to govern the entrance of a third party (i.e. non-essential) staff onto the PICU.

19.2.2. PICU staff should provide input into the organisational induction training programme.

19.2.3. Where possible, all support staff should receive full induction and training regarding the PICU environment (see 16.2.6).

19.2.4. All support staff should receive a general information pack regarding PICU services.

19.2.5. All patients should receive consistent input from support services across PICU and adult acute mental health services. In addition, some staff may require some input from forensic support services.

19.2.6. When entering the PICU environment, all external and support staff should report to the nurse in charge or shift co-ordinator to receive a handover appropriate to their presence and purpose for visiting the unit, and in accordance with local policies.

19.2.7. External staff should be asked to produce photographic identification, such as employer's identification or student card. Where such a form of identification cannot be produced, the visitor may be allowed to enter if they can be positively identified in person by a permanent member of staff within the PICU.

19.2.8. All external and support staff should be oriented to the PICU environment and informed of safety issues (e.g. security systems). They should not be issued with keys but should be issued with an alarm if entering patient areas.

19.2.9. Support staff may include legal representatives, voluntary services, maintenance staff, advocates and spiritual advisors to patients. These external staff should be asked to make appointments with the PICU in advance whenever practicable. These visitors should be allowed privacy with the patient at the discretion of the clinical team with regard for the risk assessment of the patient.

\subsection{Good practice guidance}

19.3.1. Staff essential to the operational maintenance of the PICU should be given appropriate training to understand the specialist remit of the PICU (see Chapter 16).

19.3.2 Any third party staff entering a PICU for the benefit of patients should be given relevant information and/or resources to minimise risk and maximise interaction with the patient they are there to benefit. 


\section{Other types of psychiatric intensive care}

\subsection{Rationale}

The PICU is a specialist area within inpatient mental health services; NAPICU recognises that there are developing sub-specialities within psychiatric intensive care. Whilst most of the standards within this document will be applicable, there may be need for additional guidance and integrated governance over these developing sub-specialist areas of PICU.

\subsection{Standard}

20.2.1. The standards contained within this document relate to the prevailing model of psychiatric intensive care in the UK. This model is one of an environment physically located within and part of the care pathway for general adult psychiatry and local inpatient mental health services (see Chapter 6).

20.2.2. The principles of psychiatric intensive care can be applied to services which operate with a level of security above that which is available in low secure and general adult psychiatric environments. Furthermore, in recent years, specific psychiatric intensive care services have developed for children and adolescent, and female populations.

20.2.3. The following additional types of psychiatric intensive care are not intended to be exhaustive, but give some examples of where the philosophy of psychiatric intensive care may apply.

\section{Female PICU}

20.2.4. PICUs specialising in the care and treatment of women-only are a well-recognised component of many acute adult inpatient care pathways in the UK.

20.2.5. Standards for a female PICU should follow the essence of what is contained in this document.

20.2.6. The female PICU should interface with clinical services which account for the medical and psychiatric needs of women.

20.2.7. The female PICU should be staffed with an MDT which has regard for the needs of women. The team should be skilled in the assessment and management of the mental and physical health disorders presented by women in acute inpatient psychiatry.

20.2.8. The clinical risk assessment practice in the female PICU should be sensitive to the needs of women. The management of acute and non-acute disturbance in female PICUs requires a skill-set and resource which is different to male-only or mixed settings.

20.2.9. The physical environment of the female PICU should be designed to meet the expectations and needs of women.

\section{Child er adolescent PICU}

20.2.10. PICUs specialising in the care and treatment of children and adolescents are already present in the child and adolescent inpatient care pathway in the UK. 
20.2.11. Standards for a child and adolescent PICU should follow the essence of what is contained in this document.

20.2.12. The child and adolescent PICU should interface with clinical services which account for the medical and psychiatric needs of children and adolescents.

20.2.13. The child and adolescent PICU should be staffed with an MDT which has regard for the needs of children and adolescents. The team should be skilled in the assessment and management of the mental and physical health disorders presented by children and adolescents within acute inpatient psychiatry.

20.2.14. The clinical risk assessment practice in the child and adolescent PICU should be sensitive to the needs of children and adolescents.

20.2.15. The physical environment of the child and adolescent PICU should be designed to meet the expectations and needs of children and adolescents.

20.2.16. Key aspects specific to a child and adolescent PICU include:

- Referrals into a child and adolescent PICU may cover a much broader diagnostic range than most adult PICUs. Children with associated or primary developmental disorders, long-standing challenging behaviour, incipient psychosis and emergent personality disorder will not be uncommon. The referral pathway and inclusion criteria should reflect this potential need;

- Age-specific use of the MHA 1983, Mental Capacity Act 2005 and Children Act 2004;

- The range of agencies involved in the management of children and adolescents in an acute phase of mental disorder will include those that are not routinely linked to the adult PICU. This could include educational agencies, the Family Court, children and families social services, and therapists specialising in family work;

- The nature of risk assessment, risk management, child protection/safeguarding work and criminal justice liaison should be appropriate to children and adolescents. Its level of complexity and intensity will require additional training, resources and may impact on length of stay in the PICU;

- Clinical procedures and interventions should be age-appropriate. For example, clear standards around the physical restraint of children, consultant oversight of psychopharmacological treatment plans and care-planning processes that suit the different stages of a child's development.

20.2.17. Other key standards to be read in conjunction with this document include:

- Quality Network for Inpatient CAMHS (http://www.rcpsych.ac.uk/quality/quality,accreditationaudit/ qnic1.aspx; http://www.rcpsych.ac.uk/pdf/CAMHS\%20Position\%20Statement.pdf)

- Ofsted Inspection Framework (Ofsted, 2013) (the child and adolescent PICU environment, treatment, care and outcomes may be subject to Ofsted inspection)

- Working Together to Safeguard Children (HM Government, 2013).

20.2.18. NAPICU has identified a paucity of standards within the area of child and adolescent PICUs and is anticipating the publication of national standards for child and adolescent PICUs during 2015.

\section{Medium secure PICU}

20.2.19. A medium secure PICU is a PICU within forensic services, operating at the medium secure level. These are an established part of the forensic inpatient care pathway in the UK.

20.2.20. Standards for a medium secure PICU should follow the essence of what is contained in this document.

20.2.21. Medium secure PICUs should re-formulate some of the standards contained in this document, to be applicable within the clinical and risk management environment of a medium secure inpatient setting (see 18.2.6 - 18.2.11). 
20.2.22. Key aspects specific to a medium secure PICU include:

- This patient group may have active or historical involvement with the criminal justice system, prison, probation, Ministry of Justice or other offender management organisations;

- Forensic patient populations including those in medium secure PICU are more likely to have a primary diagnosis of personality disorder alone, or co-morbid with mental illness diagnosis or diagnoses;

- Patient historical risk of violence is likely to be at the level of grievous bodily harm (GBH) and more serious offences, including offences which may have high media profile;

- There are likely to be victim issues to take into consideration in the management and care pathway of this patient group;

- There may be concurrent factors relating to the MHA 1983 status and a custodial sentence that may need to be taken into account in the care pathway;

- Certain patients subject to restriction orders under Section 41 who also have concomitant immigration problems may be under scrutiny from the Ministry of Justice or the UK Border Agency with regard to deportation on the basis of the index offence.

20.2.23. Other key standards to be read in conjunction with this document include:

- Best practice guidance: specification for adult medium secure services (Department of Health, 2007)

- Environmental design guide: adult medium secure services (Department of Health, 2011a)

- NHS standard contract for medium and low secure mental health services (adults)

- Implementation criteria for recommended specification: adult medium secure units (Tucker \& ReeveHoyland, 2011)

- See, think, act: your guide to relational security (Department of Health, 2010b)

- Standards and criteria for women in medium secure care (Ince \& Tucker, 2008)

- Standards for people with learning disabilities in medium secure care (Phillips, 2011)

- Standards for relational security (Hillier, 2011)

- Standards for deaf people in medium secure care (O'Rourke et al. 2011)

- Standards for psychotherapy in medium secure units (Jacob \& MacAllister, 2012)

- Standards for interventions to address problematic drug and alcohol use in medium security (Clark \& Sandbrook, 2013)

- National Secure Forensic Mental Health for Young People service (SFMHYPs) (http://www. specialisedservices.nhs.uk/library/36/Service Specification and Standards National Secure Forensic Adolescent Mental Health Service $M \bar{H}$ LD.pdf)

\section{High secure PICU}

20.2.24. High secure PICUs are PICUs within forensic services which operate at the high secure level. These are an established part of the forensic inpatient care pathway in the UK.

20.2.25. Standards for a high secure PICU should follow the essence of what is contained in this document.

20.2.26. High secure PICUs should re-formulate some of the standards contained in this document, to be applicable within the clinical and risk management environment of a high secure inpatient setting (see 18.2.6 - 18.2.11).

\subsubsection{Key aspects specific to high secure PICUs include:}

- Factors identified as key specific aspects to be considered for a medium secure PICU (20.2.22);

- This patient group presents with a level of risk that cannot be managed in the physical, procedural and relational security provided within a medium secure setting;

- The level of physical and procedural security within which patients are being managed is equivalent to that in place in a Category B prison; 
- Patients are likely to pose specific risks, which include evidence of:

- Planned use of weapons including knives, explosives, firearms or homemade weapons, or concealing weapons in other secure establishments;

- Serious sadistic behaviour or serious sexual assaults on staff or patients or members of the public;

- Use of poisons;

- Propensity to fire-setting which cannot be managed at lower security levels;

- Attempts or actual hostage-taking;

- Persistent, scheming or determined absconding or escape from other secure establishments.

- Certain patients are subject to 'long term segregation' (Department of Health, 2008: 15.63) and their risk to others is a constant feature of their presentation and is not subject to amelioration by a short period of seclusion combined with any other form of treatment. In addition, review schedules are individually determined by care teams as required by the MHA 1983 Code of Practice (Department of Health, 2008: 15.65) which states that that the way in which the patient's situation is reviewed should reflect the specific nature of their management plan';

- Patients may be subject to night-time confinement;

- Each high secure hospital has its own policies;

- Different types of treatment (e.g. ECT for treatment resistant schizophrenia, G-CSF for clozapine-induced neutropenia, mechanical restraint for quality of life interventions, nasogastric medication);

- Certain high secure hospitals may also have national services for highly specialised patient groups (e.g. National High Secure Deaf Service, learning disability service and women's service, all at Rampton Hospital).

20.2.28. Other key standards to be read in conjunction with this document include:

- Section 4 of the National Health Services Act 2006 (http://www.legislation.gov.uk/ukpga/2006/41/ section/4)

- High secure building design guide (Department of Health, 2010c)

- High security psychiatric services (arrangements for safety and security at Ashworth, Broadmoor and Rampton Hospitals) Directions 2011 (Department of Health, 2011b).

- The high security psychiatric services (arrangements for safety and security at Ashworth, Broadmoor and Rampton Hospitals) amendment directions 2012 (Department of Health, 2012d)

- National standards for the provision of social care services in the high security hospitals (Department of Health, 2001)

- NHS standard contract for high secure mental health services (adults)

- The high security psychiatric services (National Health Service Commissioning Board) directions 2013 (Department of Health, 2013)

- Designation framework report: national high secure male deaf services (James \& Preston, 2010).

\subsection{Good practice guidance}

20.3.1. Any PICU operating in an arena other than the conventional general adult PICU should review the standards within this document and consider which apply. The service should record a rationale where the unit needs to deviate from the prescribed standards in this document.

20.3.2. Although the essence of the standards within this document apply to all PICUs, it is self-evident that some deviation from these standards may be necessary on the grounds of age-appropriate, gender-sensitive and risk-related care or containment. 


\section{Staffing levels}

\subsection{Rationale}

Psychiatric intensive care is an integral component of the acute inpatient psychiatric care pathway, caring for the most acutely unwell and disturbed patients in psychiatric services. Such inpatients require intensive assessment and treatment during this phase of their illness; this should be delivered in the specialist environment of the PICU, by an MDT of highly skilled staff.

The provision and delivery of an effective PICU service requires sufficient and specialist support from both established staffing levels and affiliated clinical and managerial resources.

\subsection{Standard}

21.2.1. The PICU staffing should be sufficient to safely deliver optimal treatment and care, manage demand, and be responsive to critical care, 24 hours a day, 7 days a week.

21.2.2. PICUs require a comprehensive, cohesive team which share the vision for patient care in the acute clinical environment of the PICU.

21.2.3. PICUs require staff with the capacity and capability to engage effectively with highly disturbed patients with complex needs, to provide a range of multidisciplinary therapeutic interventions and clinical treatments.

21.2.4. The strength of the PICU team in delivering high quality patient care is in its full multidisciplinary nature. The MDT should include a broad range of professionals, over and above the core nursing and medical professionals. If staff of other disciplines are not directly provided for within the PICU team, there should be local contractual arrangements in place to provide these in a sustained fashion e.g. service level agreements with the local acute medical hospital for pharmacy services, contracts for psychologist input, occupational therapy input and sessional therapy staff.

21.2.5. The clinical establishment of the PICU should not be adversely decompensated to operate other local clinical services, for example the place of safety (PoS) which may be located adjacent to or attached to the PICU (see Appendix 2). Staffing for the operational running of services such as the PoS should be provided for in addition to the staffing establishment of the PICU.

\section{Nursing staff}

21.2.6. The PICU should have an agreed core establishment of nursing staff, providing a minimum staffing level across all shifts. The establishment for each PICU should be developed by registered nurses practising at the front line in conjunction with senior operational managers, and should be approved by the chief nurse with the provider organisation's board retaining responsibility (NICE 2014).

21.2.7. A nursing team consists of qualified registered specialist mental health nurses, plus unqualified nursing staff such as healthcare support workers and assistant practitioners.

21.2.8. All regular and temporary (bank and agency) nursing staff should be trained and competent in the use of breakaway techniques, the management of disturbed behaviour and the therapeutic management of violence and aggression (see Chapter 16). 
21.2.9. The actual numbers of established nursing staff posts required on a PICU will vary according to a number of factors (see Chapter 4) but will predominantly be based on the number of beds and bed occupancy. It should take into account planned and predictable variations in staff availability e.g. due to all types of leave (NICE 2014).

21.2.10. The NICE guidance for staffing levels in an acute hospital adult inpatient ward (NICE 2014) does not specify nursing staff-to-patient figures for minimum safe care, rather it emphasises the principle for local decisions that reflect the needs of the patient group. As an example, higher staffing levels are required on medical intensive care wards as compared to general medical wards (in general medical hospitals). The same principle should to be applied to psychiatric wards, and higher staffing levels are required on PICUs as compared to general psychiatric wards.

21.2.11. The following are recommended based on clinical and operational experience:

- The nursing staff establishment on PICUs should be at least one third higher than on general acute psychiatric wards (weighted per bed).

- On each shift, a third of the nursing staff should be qualified, and no less than two per shift.

- As an example, minimum shift staff numbers for ten patients in a male PICU should not fall below six for the early and late shift, and four for the night shift. These numbers should not include management and other specific therapy staff.

21.2.12. Managerial systems should be in place to ensure that all factors affecting staffing numbers and skill mix are considered and to ensure that on each shift the number of staff and their clinical expertise are sufficient to safely treat and manage the patients (see Chapters 4 and 5).

21.2.13. Staff mix and ratios should be flexible enough to meet changing levels of clinical and risk need. Staff levels should be reviewed on a daily basis. Increases or decreases in staffing depend on variable patient and staff related factors (see Chapter 4 for full details). Examples include:

- The clinical and risk acuity of individual patients' mental disorders;

- The level and array of risk being managed for individual patients (including serious vulnerabilities, serious risks to self and serious risks to others); and across the PICU's patient casemix.

- The level of use of clinical interventions which necessitate higher nursing intensity and/or observation, e.g. the level of one-to-one or greater psychiatric nursing observations, use of seclusion and ECAs.

\section{Medical staff}

21.2.14. Each PICU should have dedicated consultant psychiatrist able to function as the RC within the meaning of the MHA 1983; plus at least one single dedicated sub-consultant grade doctor (often a trainee psychiatrist) (see Chapter 4).

21.2.15. Medical staffing levels need to be determined locally based on a variety of factors, including the clinical characteristics of the patients, the size of the PICU, the acuity levels, the strength of PICU interfaces with acute wards and other services, and any associated responsibilities of the PICU consultant psychiatrist.

21.2.16. Both within routine "office hours" and out-of-hours, the PICU should have access to medical input which can respond appropriately to urgent and critical clinical scenarios. The PICU should be a responsive and dynamic service able to operate 24 hours a day, 7 days a week.

21.2.17. A doctor should be available at all times to be alerted to emergencies on the PICU. This doctor should be able to attend the PICU in a timeframe appropriate for an emergency response. Emergencies include both medical and psychiatric emergencies, e.g. to assist in the management plan for a patient(s) with acutely disturbed or violent behaviour. 
21.2.18. In addition to the core established nursing and medical staff, the PICU team must also include the following:

- Occupational therapist with specialist skills in acute psychiatric inpatient care, and support staff (e.g. activities co-ordinator). This includes working on the PICU in the evenings and weekends in order to ensure that there is a defined activity programme available to all patients available at weekends and evenings (College of Occupational Therapists 2012),

- Qualified specialist clinical psychologist for a minimum of one day per week per PICU to deliver face-toface patient care.

- Specialist mental health pharmacist who visits the PICU on a daily basis and takes part in the weekly multidisciplinary reviews; and support staff (e.g. pharmacy technicians/assistants).

- A PICU manager and a deputy, both with relevant clinical expertise in PICU.

21.2.19. It is recommended that the PICU patients have access to the following staff (this list is not exhaustive):

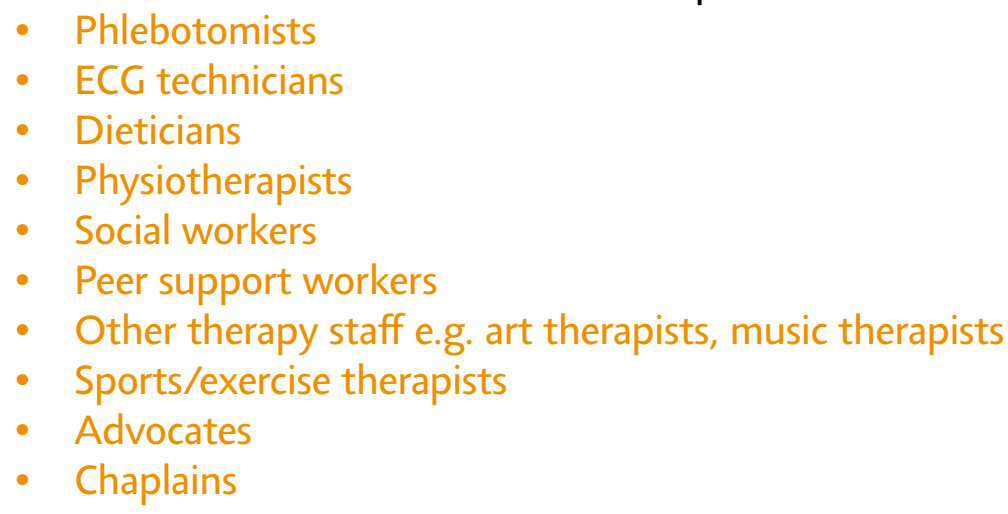

21.2.20. Students of all disciplines should be considered supernumerary.

\subsection{Good practice guidance}

21.3.1. There is no set standard for the number of PICU beds to be provided per head of population in the UK. Commissioners should use the principles outlined in this document to locally devise the level of PICU bed provision required. This will vary regionally depending on local factors. As with all clinical services, the balance between local assessment of need and the commissioned provision should be reviewed frequently from the organisational perspective e.g. at least every 3 years.

21.3.2. From a clinical and operational perspective smaller PICUs tend to function better. No more than 14 beds are recommended (see Chapter 7). Commissioners and managers should be aware that there is not a direct linear relationship between staffing numbers e.g. a 7 bedded PICU does not require half the staffing of a 14 bedded PICU. 
Bouras, N. and Holt, G. (2004) Mental health services for adults with learning disabilities. British Journal of Psychiatry 184, 291-292.

Care Quality Commission (2010) Essential standards of quality and safety.

http://www.cqc.org.uk/sites/default/files/media/documents/gac _ dec_2011 update.pdf

Care Quality Commission (2011) Count me in 2010.

http://www.cqc.org.uk/sites/default/files/media/documents/count me in 2010 final tagged.pdf

Clark, T. and Sandbrook, J. (eds) (2013) Standards for interventions to address problemtatic drug and alcohol use in medium security. Royal College of Psychiatrists Centre for Quality Improvement, CCQI 146. http://www.rcpsych.ac.uk/pdf/QNFMHS\%20Standards for_substance\%20misuse\%20interventions $\% 20$ for\%20use\%20within\%20MSUs.pdf

College of Occupational Therapists (2012). Occupational therapists' use of occupation-focused practice in secure hospitals. London: COT.

http://www.cot.co.uk/publication/practice-guidelines/occupational-therapists'-use-occupation-focusedpractice-secure-hosp

Cooper, S., Smiley, E., Morrison, J., Williamson, A. and Allan, L. (2007) Mental ill-health in adults with intellectual disabilities: prevalence and associated factors. British Journal of Psychiatry. 190, 27-35.

Cresswell, J., Hinchcliffe, G. and Lemmey, S. (2010) Accreditation for inpatient mental health services (AIMS): Standards for psychiatric intensive care units (PICUs), 2nd ed. Royal College of Psychiatrists, CRTU078. http://www.rcpsych.ac.uk/PDF/AIMS-PICU\%20Standards\%20-\%20Second\%20Edition\%20 -\%20FINAL\%20new\%20template.pdf

Deb, S., Thomas, M. \& Bright, C. (2001) Mental disorder in adults with intellectual disability: prevalence of functional psychiatric illness among a community-based population aged between 16 and 64 years. Journal of Intellectual Disability Research. 45, 495- 505.

Department of Health (1999) A national service framework for mental health: modern standards and service models.

https://www.gov.uk/government/publications/quality-standards-for-mental-health-services

Department of Health (2001) Valuing People: A New Strategy for Learning Disability for the 21st Century. https://www.gov.uk/government/uploads/system/uploads/attachment data/file/250877/5086.pdf

Department of Health (2001) National standards for the provision of social care services in the high security hospitals.

http://webarchive.nationalarchives.gov.uk/+/www.dh.gov.uk/en/Publicationsandstatistics/

Lettersandcirculars/Chiefinspectorletters/DH 4004962

Department of Health (2007) Best practice guidance: specification for adult medium-secure services.

http://webarchive.nationalarchives.gov.uk/20130107105354/http:/www.dh.gov.uk/en/

Publicationsandstatistics/Publications/PublicationsPolicyAndGuidance/DH_078744

Department of Health (2008) Code of practice: Mental Health Act 1983. TSO, London. http://www.lbhf.gov.uk/Images/Code\%20of\%20practice\%201983\%20rev\%202008\%20 dh $087073 \% 5 B 1 \% 5 D$ tcm21-145032.pdf 
Department of Health (2010a) Delivering same sex accommodation: self assessment, delivery planning and assurance.

http://webarchive.nationalarchives.gov.uk/20130107105354/http://www.dh.gov.uk/prod_consum dh/ groups/dh_digitalassets/documents/digitalasset/dh_112178.pdf

Department of Health (2010b) See, think, act: your guide to relational security. http://webarchive.nationalarchives.gov.uk/20130107105354/http://www.dh.gov.uk/en/

Publicationsandstatistics/Publications/PublicationsPolicyAndGuidance/DH 113318

Department of Health (2010c) High secure building design guide: overarching principles - for Ashworth, Broadmoor, Rampton Hospitals.

https://www.gov.uk/government/publications/high-secure-building-design-guide-overarching-principlesfor-ashworth-broadmoor-rampton-hospitals

Department of Health (2011a) Environmental design guide: adult medium secure services.

https://www.gov.uk/government/publications/environmental-design-guide-adult-medium-secureservices

Department of Health (2011b) High security psychiatric services (arrangements for safety and security at Ashworth, Broadmoor and Rampton Hospitals) Directions 2011.

https://www.gov.uk/government/publications/the-high-security-psychiatric-services-arrangements-forsafety-and-security-at-ashworth-broadmoor-and-rampton-hospitals-directions-2011

Department of Health (2012a) Psychiatric intensive care: good practice commissioning guide, consultation draft.

Department of Health (2012b) Low secure services: good practice commissioning guide, consultation draft.

Department of Health (2012c) The NHS Friends and Family Test: implementation guidance.

https://www.gov.uk/government/uploads/system/uploads/attachment data/file/127088/NHS-Friendsand-Family-Test-Implementation-Guidance-v2.pdf.pdf

Department of Health (2012d) The high security psychiatric services (arrangements for safety and security at Ashworth, Broadmoor and Rampton Hospitals) amendment directions 2012.

https://www.gov.uk/government/publications/amendments-to-the-high-security-psychiatric-servicesdirections-2012

Department of Health (2013) The high security psychiatric services (National Health Service Commissioning Board) directions 2013.

https://www.gov.uk/government/publications/high-security-psychiatric-services-national-health-servicecommissioning-board-directions-2013

Department of Health (2014) Positive and proactive care: reducing the need for restrictive interventions. https://www.gov.uk/government/uploads/system/uploads/attachment_data/file/300293/JRA_DoH Guidance on RP web accessible.pdf

Dix, R. (1995) A nurse-led psychiatric intensive care unit. Psychiatric Bulletin. 19: 258-287.

Dix, R. and Page, M. J. (2008) Physical environment. In: Psychiatric Intensive Care, 2nd edition (eds M. D.Beer, S. M. Pereira and C. Paton). Cambridge University Press, pp. 294-305.

Docking, M., Grace, K. and Bucke, T.(2008) Police custody as a 'Place of Safety': examining the use of Section 136 of the Mental Health Act 1983. IPCC Research and Statistics Series, paper 11.

http://www.ipcc.gov.uk/page/mental-health-police-custody 
Equality Act 2010: Parliament, London: Her Majesty's Stationery Office.

Hillier, B. (ed.) (2011) Standards for relational security.

http://www.rcpsych.ac.uk/pdf/Standards\%20for\%20Relational\%20Security1.pdf

HM Government (2013) Working together to safeguard children: a guide to inter-agency working to safeguard and promote the welfare of children.

http://media.education.gov.uk/assets/files/pdf/w/working\%20together.pdf

Ince, C. and Tucker, S. (eds) (2008) Standards and criteria for women in medium secure care. Royal College of Psychiatrists, CTRU 061.

http://www.rcpsych.ac.uk/PDF/Imp\%20Criteria\%20with\%20Standards\%20for\%20Women\%20Final. pdf

IPCC (2007) Forum for Preventing Deaths in Custody Annual Report 2006-2007. IPCC, FPDC/1. http://news.bbc.co.uk/1/shared/bsp/hi/pdfs/forum for_preventing_deaths_21 09 07.pdf

Jacob, C. and MacAllister, P. (eds) (2012) Standards for psychotherapy in medium secure units. Royal College for Psychiatrists Centre for Quality Improvement, CCQI 132.

http://www.rcpsych.ac.uk/PDF/Standards for_Psychotherapy in_MSUs June\%202012.pdf

James, M. and Preston, K. (2010) Designation framework report: national high secure male deaf services. Rampton Hospital.

http://www.specialisedservices.nhs.uk/library/37/High Secure Male Deaf.pdf

Lemmey, S., Bleksley, S., Cresswell, J. and Lelliott, P. (2011) Accreditation for inpatient mental health services - psychiatric intensive care units (AIMS-PICU): pilot phase report November 2009 - November 2010. Royal College of Psychiatrists, CRTU100.

NHS Executive (2000) Safety, privacy and dignity in mental health units: guidance on mixed sex accommodation for mental health services. Department of Health, pp. 26

http://webarchive.nationalarchives.gov.uk/20130107105354/http://www.dh.gov.uk/en/

Publicationsandstatistics/Publications/PublicationsPolicyAndGuidance/DH 4007095

NHS Institute for Innovation and Improvement (2012) The fifteen steps challenge.

http://www.institute.nhs.uk/productives/15stepschallenge/15stepschallenge.html

NICE (2002) Principles for best practice in clinical audit. Radcliffe Medical Press.

http://www.nice.org.uk/niceMedia/pdf/BestPracticeClinicalAudit.pdf

NICE (2003) Guidance on the use of electroconvulsive therapy. NICE, TA59.

http://guidance.nice.org.uk/TA59/Guidance/pdf/English

NICE (2005) Violence: the short term management of disturbed and violent behaviour in inpatient psychiatric settings and emergency departments. NICE, CG25.

http://guidance.nice.org.uk/CG25/NICEGuidance/pdf/English

NICE (2012) Service user experience in adult mental health: full guideline. The British Psychological Society \& The Royal College of Psychiatrists, CG136.

http://guidance.nice.org.uk/CG136/Guidance

NICE (2014) Safe staffing for nursing in adult inpatient wards in acute hospitals. NICE Safe staffing guideline 1. 
NDTi (2012) Reasonably Adjusted? Mental Health Services for People with Autism and People with Learning Disabilities. Bath: National Development Team for Inclusion.

Ofsted (2013) The framework for school inspection. http://www.ofsted.gov.uk/resources/120100

O'Rourke, S., Gibbon, S. and Hough, W (eds) (2011) Standards for deaf people in medium secure care. Royal College of Psychiatrists Centre for Quality Improvement.

http://www.rcpsych.ac.uk/PDF/Standards\%20for\%20Deaf\%20People\%20in\%20Medium\%20

Secure\%20Care.pdf

Pereira, S. and Clinton, C. (eds) (2002) Mental health policy implementation guide: national minimum standards for general adult services in psychiatric intensive care units (PICU) and low secure environments. http://webarchive.nationalarchives.gov.uk/+/www.dh.gov.uk/en/Publicationsandstatistics/Publications/ PublicationsPolicyAndGuidance/DH 4010439

Phillips, N. (ed.) (2011) Standards for people with learning disabilities in medium secure care. Royal College of Psychiatrists Centre for Quality Improvement. http://www.rcpsych.ac.uk/PDF/Standards\%20for\%20Learning\%20Disability\%20in\%20Medium\%20 Secure\%20Care\%20-\%20final.pdf

Royal College of Psychiatrists (2010) Role of the consultant psychiatrist: leadership and excellence in mental health services. Royal Society of Psychiatrists, OP74.

https://www.rcpsych.ac.uk/files/pdfversion/OP74.pdf

Royal College of Psychiatrists (2011) Standards on the use of Section 136 of the Mental Health Act 1983 (England and Wales). Royal College of Psychiatrists, CR159.

http://www.rcpsych.ac.uk/files/pdfversion/CR159x.pdf

Tucker, S. and Reeve-Hoyland, M. (2011) Implementation criteria for recommended specification: adult medium secure units, 2nd edition. Royal College of Psychiatrists Centre for Quality Improvement, CCQI 105. http://www.rcpsych.ac.uk/pdf/Implementation\%20Criteria Second\%20Edition.pdf

Waite, J. and Easton, J. (eds) (2013) The ECT handbook, 3rd edition. Royal College of Psychiatrists CR176, pp. 288.

Wagner, R. and Harter, J. K. (2006) 12: The elements of great managing. Gallup Press, pp. 237.

Wilson, B. A., Greenfield, E., Clare, L., Baddeley, A., Cockburn, J., Watson, P., Tate, R., Sopena, S., Nannery, R. and Crawford, J. (2008) Rivermead Behavioural Memory Test, 3rd edition (RBMT-3). Pearson Assessment.

Worthington, A. and Rooney, P. (2010) The Triangle of Care. Carers included: a guide to best practice in acute mental health care.

http://static.carers.org/files/caretriangle-web-5250.pdf 


\section{Acknowledgements}

NAPICU would like to thank the following individuals and professional organisations who have contributed to the development of these standards and worked tirelessly in the pursuit of high quality within psychiatric intensive care:

The working group convened to oversee the completion of these standards:

Mr Thomas Kearney

Mr Roland Dix

Dr Stephen Dye

Mr Alan Metherall

Dr Faisil Sethi

Mrs Caroline Parker

\section{Individuals}

Dr Stephen Pereira

Mr Paul Deacon

Mr Chris Dzikiti

Dr Daniel Hume

Mr Andy Johnston

Dr Aileen O'Brien

Mr Mathew Page

Mr Peter Pratt

Dr Shanika Balachandra

Mrs Ann Carrington

Dr Claire Dillon

Dr Bradley Hillier

Dr Rafik Refaat

Dr Alex Sales

Dr Clive Geoffrey Stanton

Dr Koravangattu Valsraj
NAPICU Executive (Project Lead and Lead Author)

NAPICU Editor-in-Chief (Journal)

NAPICU Executive Director of Research

NAPICU Treasurer

NAPICU Vice Chairman

NAPICU Director of Operations

NAPICU Chairman

NAPICU Executive member

NAPICU Executive Member

NAPICU Executive Member

NAPICU Executive Member

NAPICU Director of Education

NAPICU Director of Policy

NAPICU Executive member

Consultant Psychiatrist, Shannon \& Nile PICUs, St Charles Hospital, Central and North West London NHS Foundation Trust

Project Manager, CAMHS Psychiatric Intensive Care, South London and Maudsley NHS Foundation Trust

Consultant Forensic Psychiatrist, Broadmoor Hospital

Specialist Registrar ST5 in Forensic Psychiatry, South West London and St Georges Mental Health NHS Trust

Consultant Child \& Adolescent Psychiatrist, East London NHS Foundation Trust

Consultant Child \& Adolescent Psychiatrist, Tavistock \& Portman NHS Foundation Trust

Consultant Psychiatrist, Prince of Wales Hospital, Australia

Consultant Psychiatrist \& Associate Clinical Director, South London and Maudsley NHS Foundation Trust

Organisations who were consulted:

Bipolar UK

British Psychological Society

Carers UK

College of Mental Health Pharmacy

College of Occupational Therapy

Mental Health Network

MIND

National Survivor User Network (NSUNs)

Rethink

Royal College of Nursing

Royal College of Psychiatrists 


\section{Appendix 1: Staff training}

The following areas are suggested as important to PICU practice and may form the basis of formal or inhouse training.

\section{A1.1. Management \& administration}

- Legal aspects (MHA 1983 Code of Practice; Department of Health, 2008)

- Report writing/record keeping

- Ward design and organisation

- Research \& development (evidence-based practice).

\section{A1.2. Assessment}

- Use of assessment scales (e.g. HoNOS, BPRS, PANSS, LUNSERS)

- Clinical \& environmental audit

- Risk assessment \& personal and patient safety

- Assessing psychopathology.

\section{A1.3. Treatment \& care management}

- Diversity training

- Cognitive-behavioural strategies

- Maintaining security \& therapeutic use of security

- Treatment of personality disorders

- Use of medication/side-effects

- Prevention and therapeutic management of violence (including seclusion)

- Restraint (training in approved methods)

- RT

- Basic life support training (including resuscitation techniques and defibrillation)

- Observational skills

- Milieu therapy

- Care of the complex needs patient (e.g. suicidal patient, substance misuse).

\section{A1.4. Interpersonal skills}

- Counselling skills

- Communication skills

- De-escalation and models of escalation

- Debriefing following an adverse incident

- Group skills

\section{A1.5. Collaborative working}

- User \& carer involvement (active partnership)

- Team working, including negotiation \& conflict resolution skills

- Training skills (patients, carers, students, junior staff)

- Psycho-educational approaches

- Working with families (family therapy).

\section{A1.6. Other issues to consider}

- The psychological and social impact on the patient of being in a secure environment (stigma and loss of liberty)

- Working with difficult-to-engage patients

- Maintaining close and prolonged interpersonal contact with often demanding and challenging individuals (avoiding burnout)

- The impact of maintaining strict boundaries (limit-setting) within an interpersonal space which is often uncertain. 


\section{Appendix 2: Places of safety \\ (where operationally overseen by a PICU)}

\section{A2.1. Introduction}

A2.1.1. The NHS estate in relation to acute wards, PICU and Section 136 suites has developed over the last decade, due significantly to access to central funding to ensure that recommendations and improvements identified in a number of national surveys were achieved. As a result of the manner in which central funds were made available, many NHS trusts have developed new health-based places of safety (PoS) attached to and supported by newly developed (or refurbished) PICUs.

A2.1.2. As a result, a significant number of PICUs now oversee the operation of a PoS which adjoins their respective PICU. Clearly, this phenomenon requires careful planning, clinical delivery and monitoring to ensure that the safety, quality and integrity of both provisions are maintained.

A2.1.3. It is well understood that there is no clinical correlation between PICU and Section 136 of the MHA 1983, and current data would suggest that only a small number of those detained under Section 136 require direct formal admission to a PICU. However, the skill, expertise in managing challenging or disturbed behaviour and responsiveness of PICU teams coupled with the increased staffing establishments often found within PICUs have made the joint provision 'easy bed-fellows' within some organisations.

A2.1.4. For services which clinically and operationally oversee both PICU and PoS, there are considered standards which should be met to ensure the safety, quality and integrity of provision is maintained.

\section{A2.2. Current national standards}

A2.2.1. Revised national standards for Section 136 were published in July 2011 (Royal College of Psychiatrists, 2011). These standards and recommendations were developed by a multi-agency group led by the Royal College of Psychiatrists and involved agencies representing all elements of the pathway. It followed the publication of a number of influential publications including an Independent Police Complaints Commission (IPCC) study (Docking et al. 2008).

A2.2.2. All PICU staff overseeing the provision of a PoS must be cognisant of the standards within CR 159 (Royal College of Psychiatrists, 2011) and be able to demonstrate how they meet those standards pertaining to their respective part of the pathway.

A2.2.3. Specific standards that a PICU-managed PoS should demonstrably meet are summarised in A2.4.4 A2.4.10 (these are taken from CR 159; Royal College of Psychiatrists, 2011).

\section{A2.3. Staffing}

A2.3.1. There should be a clearly identified person in charge of the PoS at all times. A member of staff should be present to receive the patient on their arrival.

A2.3.2. Staffing levels should be sufficient 24 hours a day to ensure that the police can leave promptly after a handover period, even when the patient is disturbed. There should be no expectation that the police will remain until the assessment is completed, as currently happens in some places. In many areas this will require additional resources. 
A2.3.3. The PoS should ideally have dedicated staffing, or at the very least, a supernumerary post attached to the team responsible for the PoS.

\section{A2.4. Physical standards of the PoS}

A2.4.1. The following guidance is compliant with NICE guidance on short-term management of disturbed or violent behaviour in inpatient psychiatric settings and emergency departments (NICE, 2005).

A2.4.2. The annual report 2006-2007 for the Forum for Preventing Deaths in Custody (IPCC, 2007) highlighted the importance of the physical environment.

A2.4.3. The PoS must be a locked facility in order to be able to safely care for those who are disturbed. Levels of staff required to support this facility, when in use, are up to three staff trained in physical intervention, who should be available at short notice without compromising staffing levels and hence safety elsewhere. This is in addition to the staff carrying out the assessment. The patient's belongings should be recorded and kept in a safe place.

\section{Assessment room}

A2.4.4. The assessment room must:

- Be large enough to accommodate six people, to be able to both assess and restrain where necessary;

- Be well-lit and have an observation window;

- Have good exits, with consideration being given to there being two doors at opposite ends of the room; the doors should open outwards for the safety of staff;

- Have fixed, soft, comfortable chairs in a washable fabric; furniture and fittings should be chosen so they cannot be used to cause injury by offering a weapon of opportunity;

- Have a clock visible to both staff and the patient;

- Have no ligature points;

- Have good communication with others through a phone line with outside dialling;

- Have a panic alarm system;

- Be located near to other staff and be easily accessed by a team trained in physical intervention and the use of resuscitation equipment;

- Have CCTV to enhance safety and security;

- Have access to resuscitation equipment including a defibrillator.

\section{Supporting facilities}

A2.4.5. The patient may need to remain in the assessment unit for several hours (the MHA 1983 allows detention for up to 72 hours) although it is envisaged that someone would only be detained for prolonged periods in the most exceptional circumstances. The assessment may be delayed, for example when it is unclear whether a person under the influence of drugs and alcohol also has a mental disorder or where the need to administer emergency sedation makes the subsequent assessment impossible until the effects of the medication have subsided. It may take time to gather the relevant information and a person might be more vulnerable if discharged in the night. The assessment unit should be located in an assessment area which has the following:

- A couch for sleeping or resting and to assist any necessary medical examination;

- Saliva substance misuse screening or drug urine testing kits;

- Washing and toileting facilities;

- Decontamination facilities to remove CS spray and other noxious substances;

Provision of beverages and light snacks; 
- A drug cupboard, where the facility is in a healthcare setting;

- A place for writing up notes and briefing assessment unit staff by those involved in the detention;

- If in a hospital, a computer linked to the electronic care system to identify relevant background information, current status under the MHA 1983, crisis plans, advanced statements or decisions;

- Electronic copies of patient leaflets in less commonly used languages and formats, where they are not otherwise immediately available;

- A telephone with outside dialling arrangements;

- Facilities for carers and legal representatives, including a separate waiting area for them;

- Access to photocopying facilities;

- A written local operational procedure for the use of the assessment facility.

\section{Location of the place of safety}

A2.4.6. The unit should:

- Be accessible to the disabled and should preferably be on the ground floor;

- Be in a quiet area as the patient may need to rest;

- Have discrete access avoiding public areas;

- Be in a secure area, permitting the individual to wander or pace and, if appropriate, talk to their carers and friends;

- Have lockable sections if more than one patient is likely to require the facility at any one time, so that individuals can be kept apart.

\section{Smoking in the PoS}

A2.4.7. Many individuals detained under Section 136 will be smokers and their distress and level of disturbance may be increased by being unable to smoke. The key aim should be to ensure that their length of stay in the PoS is kept to a minimum. Due to safety considerations in the assessment period it is unlikely that a safe external space within the suite could be provided to permit smoking. Therefore it is envisaged that those detained will be unable to smoke. However, some organisations permit smoking by patients on their properties and therefore, equity should be provided for all patients.

\section{Treatment under Section 136}

A2.4.8. Section 136 does not authorise medical treatment. Medical treatment may be given with the patient's capacitous consent or, if the patient lacks capacity in relation to treatment decisions, it may be given in line with the authority of the Mental Capacity Act 2005 (http://www.legislation.gov.uk/ukpga/2005/9/ contents) and would have to be in the patient's best interest.

\section{Transport home following MHA assessment}

A2.4.9. The unit should hold a specific budget to arrange onward transport home following MHA 1983 assessment.

\section{A2.5. Monitoring}

A2.5.1. The local multi agency implementation group must ensure that there are adequate approved mental health professionals and doctors approved under Section 12 of the MHA 1983 to enable joint assessments to begin within three hours currently, with an expectation that in the longer term the target will become 
two hours.

A2.5.2. Physical health screens or assessments must be carried out on arrival at the PoS to ensure that patients are physically well enough to be detained there. If significant concerns of a physical nature arise, a transfer to the local emergency department should be considered.

A2.5.3. The CQC has responsibility for the monitoring the standard of PoS in hospitals in England and the Health Inspectorate Wales has a similar function in Wales.

\section{A2.6. Monitoring and audit}

In the absence of a statutory monitoring form, the form contained within CR 159 (Royal College of Psychiatrists, 2011) should be used for minimum data collection. Additional clinical audits should include the outcome of the detention and whether the patient:

- Was in possession of a weapon;

- Was significantly intoxicated with alcohol or substances;

- Was suspected of committing a crime;

- Was conveyed in an ambulance;

- Had a physical health screen completed;

- Received any medication;

- Had pre-existing physical health issues and were they addressed.

The above list is not exhaustive. 


\section{篓 napicu}

national association of psychiatric intensive care $\varepsilon$ low secure units

NAPICU Administrative Office

Glenfinnan Suite, Braeview House,

9/11 Braeview Place, East Kilbride,

Glasgow G74 3XH

NAPICU Administration Office Tel: 01355244585

Fax: 01355249959

Email: info@napicu.org.uk

Website: www.napicu.org.uk 